\title{
3d deconfinement, product gauge group, Seiberg-Witten and new 3d dualities
}

\author{
Keita Nii \\ Harish-Chandra Research Institute, \\ Chhatnag Road, Jhusi, Allahabad 211019, India \\ E-mail: keitanii@hri.res.in
}

ABSTRACT: We construct a three-dimensional deconfinement method which enables us to find new three-dimensional dualities and we apply various techniques developed in the fourdimensional supersymmetric gauge theories, such as product gauge groups and SeibergWitten curves, to the three-dimensional $\mathcal{N}=2$ supersymmetric gauge theories. The dual descriptions of the three-dimensional $\mathcal{N}=2$ supersymmetric gauge theories which involve two-index matters, for example, adjoint, symmetric, and anti-symmetric matters without a superpotential can be obtained. These matters are described in terms of the s-confining phases of the supersymmetric gauge theories.

Keywords: Duality in Gauge Field Theories, Supersymmetric gauge theory, Supersymmetry and Duality, Field Theories in Lower Dimensions

ARXiv EPrint: 1603.08550 


\section{Contents}

1 Introduction 1

2 Quantum aspects of $3 \mathrm{~d} \mathcal{N}=2 \operatorname{Sp}(2 N)$ and $\mathrm{SU}(N)$ gauge theories $\quad 4$

3 Deconfinement with s-confining $\times$ s-confining $\quad 6$

$\begin{array}{lll}3.1 \mathrm{SU}(2 N) & 6\end{array}$

$\begin{array}{lll}3.2 & \mathrm{SU}(2 N+1) & 14\end{array}$

$\begin{array}{lll}3.3 & \mathrm{SU}(2 N) \text { with an anti-symmetric flavor } & 16\end{array}$

4 Gauge theories with $\mathrm{SU}(2)^{N}$ gauge group $\quad 21$

4.1 Bi-fundamental and fundamental matters 22

$\begin{array}{lll}4.2 & \text { Bi-fundamental matters } & 23\end{array}$

$\begin{array}{lll}4.2 .1 \quad F=1 & 24\end{array}$

$\begin{array}{lll}4.2 .2 \quad F=2 & 24\end{array}$

$\begin{array}{lll}4.3 & \mathrm{SU}(2) \times \mathrm{SU}(2) \times \mathrm{SU}(2) & 26\end{array}$

$\begin{array}{lll}5 & \mathrm{SU}(N) \text { with an antisymmetric matter } & \mathbf{2 8}\end{array}$

5.1 Lifting up the Coulomb moduli 28

$\begin{array}{lll}5.1 .1 & \text { The electric theory } & 28\end{array}$

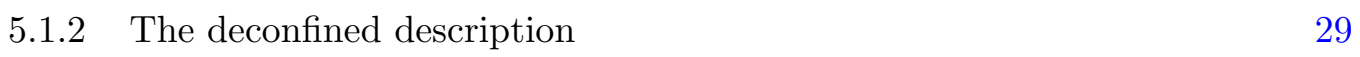

$\begin{array}{lll}5.1 .3 & \text { The first dual description } & 29\end{array}$

5.1.4 The second dual description 31

5.2 Decoupling the Coulomb moduli 34

$\begin{array}{lll}6 & \text { Summary and discussion } & 36\end{array}$

\section{Introduction}

Supersymmetry is one of the candidates for beyond the Standard Model and attracting attention as the playground for testing the various ideas of analyzing the strongly-coupled or non-perturbative dynamics, for instance, such as QCD. The supersymmetry severely constrains the perturbative corrections and we can make non-perturbative predictions by combining the holomorphic properties, the symmetry argument and the selection rules. About twenty years ago, in a series of analyses of the four-dimensional $\mathcal{N}=1$ supersymmetric gauge theories, Seiberg found a beautiful duality which relates the theories with different ranks of the gauge groups [1]. After that, various dualities which contain not only the (anti-)fundamental matters but also the adjoint, symmetric or anti-symmetric matters were found by many authors (for example, see [2] which includes the comprehensive report on the extension of the Seiberg duality). 
Among these dualities, one of the most constructive ways of finding the dualities is a so-called "deconfinement technique" [3-5], where the two-index matters, such as the anti-symmetric matters, are considered as the mesons of some (s-)confining gauge theories. Since the deconfined description only contains the fields in the (anti-)fundamental representations, the usual Seiberg duality [1] can be adapted. Thus the dual description of the $\mathrm{SU}(N)$ gauge theories with a two-index matter is given by the theories with a product of the gauge groups, $\mathrm{SU}(N) \times$ (other gauge groups). If the dual description also has the two-index matters, we can again deconfine it by additional gauge groups. In this way, we generally have the infinite duality sequences.

In three space-time dimensions, the dynamics of the supersymmetric gauge theories with four supercharges was analyzed (for example, see [6-8]), where it was found that the phenomena similar to $4 \mathrm{~d} \mathcal{N}=1$ SQCD arise: for example, chiral symmetry breaking, confinement, dynamical supersymmetry breaking. In addition, 3d SUSY gauge theories have new features, say, Coulomb branch dynamics and real masses, which is closely related to the fact that the $3 \mathrm{~d}$ vector superfield supplies the Coulomb branch. These new ingredients make the $3 \mathrm{~d}$ dynamics highly interesting and complicated.

It is now known that there are various dualities also in 3d: the Giveon-Kutasov duality [9] relates the supersymmetric Chern-Simons matter gauge theories, which is a generalization of the level-rank duality in the pure Chern-Simons theory. The Aharony duality [10] is a Seiberg-like duality of the $\mathrm{U}(N)$ or $\operatorname{Sp}(2 N)$ gauge theories without Chern-Simons terms. Furthermore the extensions including adjoint matters are very well studied [11-14]. However the deconfinement technique in $3 \mathrm{~d}$ has not been investigated yet.

Recently dynamics of the Coulomb branch in the $3 \mathrm{~d} \mathcal{N}=2$ SUSY gauge theories have been extensively studied [15-17], where it was argued that the effects of the real masses for the matter fields, of the induced Chern-Simons levels and of the Fayet-Iliopoulos terms are important to determine the low-energy dynamics. This understanding of the Coulomb branch dynamics enables us to find the relationship between the $4 \mathrm{~d}$ Seiberg dualities and the $3 \mathrm{~d}$ ones $[17,18]$.

For the 3d dualities with two-index matters, we have the $3 \mathrm{~d}$ version of the KutasovSchwimmer duality $[13,14]$. The electric side is an $\mathrm{U}\left(N_{c}\right)$ gauge theories with $N_{f}$ (anti)fundamental matters $Q, \tilde{Q}$ and an adjoint matter $X$ with a superpotential $W=\operatorname{tr} X^{k+1}$. The magnetic dual is given by an $\mathrm{U}\left(k N_{f}-N_{c}\right)$ gauge theory. The Kutasov-Schwimmer duality is regarded as the generalization of the conventional Seiberg duality containing only the fundamental matters because the superpotential with some perturbations $W=\sum_{i=1}^{k+1} g_{i} \operatorname{tr} X^{i}$ breaks the $\mathrm{U}\left(N_{c}\right)$ gauge symmetry into $\mathrm{U}\left(n_{1}\right) \times \cdots \times \mathrm{U}\left(n_{k}\right)$ and each $\mathrm{U}\left(n_{i}\right)$ part has only the fundamental matters. Thus the problem reduces to the conventional Seiberg duality at least in these perturbations. In addition, the superpotential truncates the chiral ring up to $\operatorname{tr} X^{k}$ and as the result the moduli space of vacua becomes tractable. To find the duality with two-index matters and without any superpotential is extremely non-trivial. In four-dimensions we can use the deconfinement technique to find such a duality [3-5]. However such a technique has not been developed in three-dimensions. 
In this paper we will investigate the deconfinement technique for the $3 \mathrm{~d} \mathcal{N}=2$ supersymmetric theories with two-index matters. We will especially focus on the deconfinement of the anti-symmetric matters, which is performed by the s-confining phase of the $3 \mathrm{~d}$ $\mathcal{N}=2 \operatorname{Sp}(2 N)$ gauge theory with $2 N+2$ fundamental matters. However this method is not limited to the anti-symmetrics. It will be easily generalized to the theories with the symmetric or adjoint matters. By using the deconfinement technique we will find that the large class of the $\mathrm{SU}(N)$ gauge theories with one anti-symmetric is completely s-confining. Especially, when the dynamics of the both gauge groups are s-confining, we will be able to find the non-gauge description as the dual theory. We will call this condition "s-confining $\times$ s-confining".

When considering the deconfinement in $3 \mathrm{~d}$, we are forced to study the product gauge groups $^{1}$ and the structure of their Coulomb branches since under the duality transformation the Coulomb branch will be non-trivially mapped. The rigorous proof of the mapping is quite difficult due to the strong dynamics and will be checked only from the symmetry and consistency argument. Thus we will consider the $\mathrm{SU}(2)^{N}$ product gauge theory as a supporting example on this subtlety, which is also the generalization of the "s-confining $\times$ s-confining" structure. This theory was already investigated in $4 \mathrm{~d}$ [21-23] and these authors claimed that one can derive the exact superpotential by using the holomorphy and the so-called "linearity principle" (integrating-in method) and that if we include the bi-fundamental matters, the theory is in the Coulomb phase and the $\mathcal{N}=1$ Seiberg-Witten curve can be studied. We will analyze the $3 \mathrm{~d}$ version by using the quantum dynamics of the $3 \mathrm{~d} \mathcal{N}=2 \mathrm{SU}(2)$ gauge theory and we will find the consistency with the $4 \mathrm{~d}$ exact superpotential and the dimensional reduction of the Seiberg-Witten curve [24]. As a by-product, this will support the mapping rule of the Coulomb branch under the duality transformation.

The organization of this paper is as follows. In section 2 , we will briefly review the quantum dynamics of the $3 \mathrm{~d} \mathcal{N}=2 \mathrm{Sp}(2 N)$ and $\mathrm{SU}(N)$ gauge theories which will be employed for deconfining the anti-symmetric matters. Depending on the number of (fundamental) flavors, there are various phases. In section 3 , we will show the deconfinement of a $3 \mathrm{~d} \mathcal{N}=2 \mathrm{SU}(N)$ gauge theory with anti-symmetric matters and (anti-) fundamentals. First example is a chiral gauge theory with a single anti-symmetric matter, two fundamentals and $2 N$ anti-fundamentals. Next we will study the $3 \mathrm{~d} \mathcal{N}=2 \mathrm{SU}(N)$ gauge theory with two anti-symmetric matters and the vector-like matters in a fundamental representation, which was considered by [25]. These examples are all s-confining and the dual theories are given by the non-gauge theories. The s-confining phase of the first example is a new result. In section 4, we will study the product gauge group $\mathrm{SU}(2)^{N}$, which is generalizing the "s-confining $\times$ s-confining" structure in section 3 . From the comparison with the $4 \mathrm{~d}$ exact result, we will find that the mapping rule of the Coulomb branch is correct. We will also comment on the relation between the Seiberg-Witten curve and the $3 \mathrm{~d}$ exact superpotential. In section 5 , a $3 \mathrm{~d} \mathcal{N}=2 \mathrm{SU}(N)$ gauge theory with an anti-symmetric matter and the generic number of the (anti-)fundamentals is considered. Finally in section 6 we conclude and discuss the future directions.

\footnotetext{
${ }^{1}$ The generic product gauge theories in $3 \mathrm{~d}$ were investigated in $[19,20]$ by using the brane construction.
} 


\section{Quantum aspects of $3 \mathrm{~d} \mathcal{N}=2 \operatorname{Sp}(2 N)$ and $\mathrm{SU}(N)$ gauge theories}

In this section we will briefly summarize the quantum aspects of the $3 \mathrm{~d} \mathcal{N}=2$ gauge theories with $\mathrm{SU}(N)$ or $\mathrm{Sp}(2 N)$ gauge symmetries, ${ }^{2}$ paying attention to the flat directions (moduli) of the scalar fields, which is based on [7, 26]. Since there is a $3 \mathrm{~d} \mathcal{N}=2$ supersymmetry which means the theory has four supercharges, the theory is obtained by the dimensional reduction of the $4 \mathrm{~d} \mathcal{N}=1$ supersymmetric gauge theory. Thus a $3 \mathrm{~d}$ chiral superfield contains a single complex scalar field as usual. If there is no superpotential and no quantum correction which makes this scalar massive, then the scalar field becomes a flat direction. We will call this flat direction Higgs branch.

For a vector superfield there is also a real scalar field in the adjoint representation coming from the compactified direction of the $4 \mathrm{~d}$ gauge field $A_{3}$ which is denoted as $\phi$. We usually diagonalize $\phi$ by the gauge and Weyl transformations

$$
\phi= \begin{cases}\operatorname{diag}\left(\phi_{1}, \phi_{2}, \cdots, \phi_{N}\right), \quad \phi_{1}>\phi_{2}>\cdots>\phi_{N}, \quad \sum_{i=1}^{N} \phi_{i}=0 & \text { for } \operatorname{SU}(N) \\ \operatorname{diag}\left(\phi_{1}, \cdots, \phi_{N},-\phi_{N}, \cdots,-\phi_{1}\right), \quad \phi_{1}>\phi_{2}>\cdots>\phi_{N} & \text { for } \operatorname{Sp}(2 N) .\end{cases}
$$

When this adjoint scalar $\phi$ takes a generic vev $\langle\phi\rangle \neq 0$, the gauge group is broken to $\mathrm{U}(1)^{\operatorname{rank}(G)}$, where $G$ is $\mathrm{SU}(N)$ or $\operatorname{Sp}(2 N)$. Since a three-dimensional photon is dual to a compact scalar field $\sigma$ which we usually call "dual photon", combining these two real scalars, each $\mathrm{U}(1)$ vector superfield gives one complex scalar field

$$
\Phi_{i}=\phi_{i}+i \sigma_{i}
$$

Thus the vector superfield has the $\operatorname{rank}(G)$ dimensional flat directions at the tree-level. However these directions are in many cases lifted by the instanton corrections [6-8]. In the presence of the fundamental matters, only the single branch is un-lifted, which is denoted by

$$
Y \sim \begin{cases}\exp \left(\Phi_{1}-\Phi_{N}\right) & \text { for } \operatorname{SU}(N) \\ \exp \left(\Phi_{1}+\Phi_{N}\right) & \text { for } \operatorname{Sp}(2 N)\end{cases}
$$

This exponential form is due to the fact that the dual photon is actually the compact scalar. When the above Coulomb branch operators take some vev's, the unbroken U(1) gauge symmetry introduces a topological $\mathrm{U}(1)_{J}$ symmetry whose current is defined as

$$
J_{\mathrm{U}(1)_{J}}^{\mu}=\epsilon^{\mu \nu \rho} F_{\nu \rho}^{\mathrm{U}(1)} .
$$

Since this current generates the shift symmetry of the dual photon, the Coulomb branch operator is charged under the $\mathrm{U}(1)_{J}$ global symmetry. When we usually have other $\mathrm{U}(1)$ global symmetries, say, $\mathrm{U}(1)_{B}$ which rotates the chiral superfields, the mixed Chern-Simons term between $\mathrm{U}(1)_{J}$ and $\mathrm{U}(1)_{B}$ is induced from the one-loop diagrams and leads to the mixing of the global $\mathrm{U}(1)$ symmetries in a non-trivial way. As the result, $Y$ is not charged under the $\mathrm{U}(1)_{B}$ classically but quantum mechanically charged. These mixings are calculated by the Callias index theorem [27-29]. This phenomenon has the completely identical

\footnotetext{
${ }^{2}$ The convention of the $\mathrm{Sp}(2 N)$ group is that $\mathrm{Sp}(2) \sim \mathrm{SU}(2)$
} 
origin as the fact that in the Chern-Simons theory the electrically charged particles are propagating with the magnetic flux accompanied.

Here we will show the global charges of these flat directions and the s-confining phases which we will use later. Let us first consider the $3 \mathrm{~d} \mathcal{N}=2 \mathrm{SU}(N)$ gauge theory with $F$ fundamentals $Q$ and $F$ anti-fundamentals $\tilde{Q}$. The global symmetry of the theory is $\mathrm{SU}(F)_{L} \times \mathrm{SU}(F)_{R} \times \mathrm{U}(1)_{B} \times \mathrm{U}(1)_{A} \times \mathrm{U}(1)_{R}$. The quantum numbers of the matter contents and the Coulomb branch operator are in table 1, where $\lambda$ is a gaugino.

\begin{tabular}{|c|c|c|c|c|c|c|}
\hline & $\mathrm{SU}(N)$ & $\mathrm{SU}(F)_{L}$ & $\mathrm{SU}(F)_{R}$ & $\mathrm{U}(1)_{B}$ & $\mathrm{U}(1)_{A}$ & $\mathrm{U}(1)_{R}$ \\
\hline$Q$ & $\square$ & $\square$ & 1 & 1 & 1 & $R$ \\
$\tilde{Q}$ & $\square$ & 1 & $\square$ & -1 & 1 & $R$ \\
$\lambda$ & adj. & 1 & 1 & 0 & 0 & 1 \\
\hline$Y_{\mathrm{SU}(N)}$ & 1 & 1 & 1 & 0 & $-2 F$ & $-2 F(R-1)-2(N-1)$ \\
\hline
\end{tabular}

Table 1. Quantum numbers of the electric theory.

The monopole-instanton lifts almost all the Coulomb branch and the one complex dimension is expected to be un-lifted. We call it $Y_{\mathrm{SU}(N)}$. For $F=N$, the theory is in the s-confining phase [7]. The effective degrees of freedom are a meson $M:=\bar{Q} Q$, two baryonic operators $B:=Q^{N}, \bar{B}:=\bar{Q}^{N}$ and the Coulomb branch operator $Y_{\mathrm{SU}(N)}$. The dynamics of the s-confining phase is described by a superpotential

$$
W=-Y_{\mathrm{SU}(N)}(\operatorname{det} M-B \bar{B})
$$

which is consistent with the global symmetries listed above.

For a $3 \mathrm{~d} \mathcal{N}=2 \operatorname{Sp}(2 N)$ theory with $2 F$ fundamental matters, the quantum numbers of the matter fields and the Coulomb branch operator are summarized in table 2 .

\begin{tabular}{|c|c|c|c|c|}
\hline & $\mathrm{Sp}(2 N)$ & $\mathrm{SU}(2 F)$ & $\mathrm{U}(1)_{B}$ & $\mathrm{U}(1)_{R}$ \\
\hline$Q$ & $\square$ & $\square$ & 1 & $R$ \\
$\lambda$ & adj. & 1 & 0 & 1 \\
\hline$Y_{\mathrm{Sp}(2 N)}$ & 1 & 1 & $-2 F$ & $-2 F(R-1)-2 N$ \\
\hline
\end{tabular}

Table 2. Quantum numbers of the $3 \mathrm{~d} \mathcal{N}=2 \operatorname{Sp}(2 N)$ gauge theory.

We expect that the monopole-instantons again lift most of the Coulomb branch and the complex one-dimensional part remains un-lifted and it is denoted by $Y_{\mathrm{Sp}(2 N)}$. The s-confining phase arises for $F=N+1$ [26], in which the gauge singlets $M:=Q Q$ and $Y_{\mathrm{Sp}(2 N)}$ with a superpotential

$$
W=-Y_{\mathrm{Sp}(2 N)} \operatorname{Pf} M
$$

give the correct description of the low-energy dynamics. 


\section{Deconfinement with s-confining $\times$ s-confining}

\section{$3.1 \mathrm{SU}(2 N)$}

As an illustration of how the $3 \mathrm{~d}$ deconfinement technique works, we will first consider the $3 \mathrm{~d} \mathcal{N}=2 \mathrm{SU}(2 N)$ gauge theories with a single anti-symmetric matter and some non-vector like ("chiral") matters in the (anti-)fundamental representations. In 4d, the anti-symmetric matters are deconfined into the (bi-)fundamental quarks by regarding the anti-symmetric matters as the mesons of the s-confining Sp gauge theory [3-5]. We can apply the same technique to the $3 \mathrm{~d}$ case more easily as follows. Since we have no chiral anomaly in $3 \mathrm{~d}$, without bothering about the chiral anomaly matching between the confined and deconfined theories, we can easily deconfine the anti-symmetric matters by using the s-confining phase of the $3 \mathrm{~d} \mathcal{N}=2 \operatorname{Sp}(2 N-2)$ theory. Only the subtlety comes from the existence of the Coulomb branch which will be lifted by introducing the additional singlet and by coupling it to the Coulomb branch. In this subsection, we will consider the case with the $\mathrm{SU}(2 N)$ gauge group. Next subsection, the $\mathrm{SU}(2 N+1)$ case will be given.

To be more specific, the first example is a $3 \mathrm{~d} \mathcal{N}=2 \mathrm{SU}(2 N)$ gauge theory with two fundamentals, $2 N$ anti-fundamentals and one anti-symmetric matter. We call this theory "electric". The deconfined description is easily obtained by a $\mathrm{SU}(2 N) \times \operatorname{Sp}(2 N-2)$ theory. The global symmetry of the theory is $\mathrm{SU}(2 N) \times \mathrm{SU}(2) \times \mathrm{U}(1)^{3} \times \mathrm{U}(1)_{R}$. The matter contents and their quantum numbers of the deconfined description are summarized in table 3. In the table, $Y_{\mathrm{SU}(2 N)}$ and $Y_{\mathrm{Sp}(2 N-2)}$ are the Coulomb branch coordinates of the $\mathrm{SU}(2 N)$ and $\mathrm{Sp}(2 N-2)$ gauge groups respectively and we here chose the $\mathrm{U}(1)_{R}$ charge as a generic value because we do not know the correct $\mathrm{U}(1)_{R}$ charge which is realized in the far infrared region.

\begin{tabular}{|c|c|c|c|c|c|c|c|c|}
\hline & $\mathrm{SU}(2 N)$ & $\mathrm{Sp}(2 N-2)$ & $\mathrm{SU}(2 N)$ & $\mathrm{SU}(2)$ & $\mathrm{U}(1)$ & $\mathrm{U}(1)$ & $\mathrm{U}(1)$ & $\mathrm{U}(1)_{R}$ \\
\hline$Q$ & $\square$ & $\square$ & 1 & 1 & 1 & 0 & 0 & $R_{1}$ \\
$Q^{\prime \prime}$ & $\square$ & 1 & 1 & $\square$ & 0 & 1 & 0 & $R_{2}$ \\
$\bar{Q}$ & $\square$ & 1 & $\square$ & 1 & 0 & 0 & 1 & $R_{3}$ \\
$\mathrm{~S}$ & 1 & 1 & 1 & 1 & $2 N$ & 0 & 0 & $2 N R_{1}$ \\
\hline$Y_{\mathrm{SU}(2 N)}$ & 1 & 1 & 1 & 1 & $-2 N+2$ & -2 & $-2 N$ & $-(2 N-2) R_{1}-2 R_{2}-2 N R_{3}+2$ \\
$Y_{\mathrm{Sp}(2 N-2)}$ & 1 & 1 & 1 & 1 & $-2 N$ & 0 & 0 & $-2 N R_{1}+2$ \\
\hline
\end{tabular}

Table 3. Quantum numbers of the s-confining $\times$ s-confining theory.

Due to the special number of the (anti-)fundamentals, both of the gauge groups, $\mathrm{SU}(2 N)$ and $\mathrm{Sp}(2 N-2)$ are in the s-confining phases. We call this structure "s-confining $\times$ s-confining" according to the $4 \mathrm{~d}$ case in [30] where the $4 \mathrm{~d}$ versions of "s-confining $\times$ s-confining" were considered. In this theory we can replace both of the gauge dynamics with the descriptions with only the chiral superfields and without the gauge fields. The Coulomb branch of the $\operatorname{Sp}(2 N-2)$ gauge theory is lifted by the singlet field $S$ giving the mass term

$$
W=S Y_{\mathrm{Sp}(2 N-2)}
$$


Since the dynamics of the $\operatorname{Sp}(2 N-2)$ gauge theory is in the s-confining phase, we can first replace the $\operatorname{Sp}(2 N)$ dynamics to a theory with only the $\operatorname{Sp}(2 N-2)$-gauge singlets by assuming $g_{\mathrm{SU}}^{2} \ll g_{\mathrm{Sp}}^{2}$. The low-energy effective theory becomes a $3 \mathrm{~d} \mathcal{N}=2 \mathrm{SU}(2 N)$ gauge theory in table 4 . The matter contents includes the anti-symmetric matter which we wanted to deconfine.

\begin{tabular}{|c|c|c|c|c|c|c|c|}
\hline & $\mathrm{SU}(2 N)$ & $\mathrm{SU}(2 N)$ & $\mathrm{SU}(2)$ & $\mathrm{U}(1)$ & $\mathrm{U}(1)$ & $\mathrm{U}(1)$ & $\mathrm{U}(1)_{R}$ \\
\hline$V:=Q Q$ & $\square$ & 1 & 1 & 2 & 0 & 0 & $2 R_{1}$ \\
$Q^{\prime \prime}$ & $\square$ & 1 & $\square$ & 0 & 1 & 0 & $R_{2}$ \\
$\bar{Q}$ & $\square$ & $\square$ & 1 & 0 & 0 & 1 & $R_{3}$ \\
$Y_{\mathrm{Sp}(2 N-2)}$ & 1 & 1 & 1 & $-2 N$ & 0 & 0 & $-2 N R_{1}+2$ \\
$S$ & 1 & 1 & 1 & $2 N$ & 0 & 0 & $2 N R_{1}$ \\
\hline
\end{tabular}

Table 4. Quantum numbers of the electric $\mathrm{SU}(2 N)$ theory.

The superpotential describing the s-confining phase of the $\operatorname{Sp}(2 N-2)$ gauge theory is

$$
W=-Y_{\mathrm{Sp}(2 N-2)} V^{N}+S Y_{\mathrm{Sp}(2 N-2)},
$$

where the notations like $V^{N}$ is a bit sloppy because more rigorously we should regard this as a Pfaffian. But in this article this abbreviation is enough for our purposes. Notice that the fields $V$ and $Y_{\mathrm{Sp}(2 N-2)}$ are not composites but elementary fields from the low-energy $\mathrm{SU}(2 N)$ gauge theory point of view since we are in the s-confining phase of the $\operatorname{Sp}(2 N)$ theory. Due to the mass term between $S$ and $Y_{\mathrm{Sp}(2 N-2)}$, the first term plays no role at all and the equation of motion for $Y_{\mathrm{Sp}(2 N-2)}$ gives $S=V^{N}$. At the low-energy limit, we have the $3 \mathrm{~d} \mathcal{N}=2 \mathrm{SU}(2 N)$ gauge theory with two fundamentals, $2 N$ anti-fundamentals and one anti-symmetric matter with no tree-level superpotential.

The Coulomb branch of this theory is complicated due to the reason discussed in $[25,31]$, where it was shown that the presence of the anti-symmetric matter and the "chiral" nature of the (anti-) fundamental matters make the Coulomb branch complicated since the various mixed Chern-Simons terms and the FI terms are effectively generated along the Coulomb branch and this leads to the modification of the monopole-instanton structure and it makes the Coulomb branch non-gauge invariant. This may allow us to have the two-dimensional Coulomb branch. Then in this case we also expect more than one coordinate to be required for describing the entire Coulomb branch. The Coulomb branch direction

$$
Y \sim\left(\begin{array}{lllll}
\sigma & & & \\
& 0 & & & \\
& & \ddots & & \\
& & & 0 & \\
& & & & -\sigma
\end{array}\right)
$$

which is usually globally defined and un-lifted should be dressed by some matter fields. This is because the chirality of the matter contents induces the effective Chern-Simons term 
along the direction $Y$, and then $Y$ becomes non-gauge invariant. The dressed Coulomb branch operators which are expected to be un-lifted are as follows.

$$
Y V^{N-1}, \quad Y V^{N-2} Q^{\prime 2}
$$

The global charges of these operators are in table 5 .

\begin{tabular}{|c|c|c|c|c|c|c|}
\hline & $\mathrm{SU}(2 N)$ & $\mathrm{SU}(2)$ & $\mathrm{U}(1)$ & $\mathrm{U}(1)$ & $\mathrm{U}(1)$ & $\mathrm{U}(1)_{R}$ \\
\hline$Y$ & 1 & 1 & $-4 N+4$ & -2 & $-2 N$ & $-(4 N-4) R_{1}-2 R_{2}-2 N R_{3}+2$ \\
$Y V^{N-1}$ & 1 & 1 & $-2 N+2$ & -2 & $-2 N$ & $-(2 N-2) R_{1}-2 R_{2}-2 N R_{3}+2$ \\
$Y V^{N-2} Q^{\prime \prime 2}$ & 1 & 1 & $-2 N$ & 0 & $-2 N$ & $-2 N R_{1}-2 N R_{3}+2$ \\
\hline
\end{tabular}

Table 5. Global symmetries of the (dressed) monopole operators.

Here we will briefly show that these composite operators are indeed gauge invariant according to the discussion in [25, 31]. First, along the Coulomb branch direction $Y$, the gauge group is broken as

$$
\mathrm{SU}(2 N) \rightarrow \mathrm{SU}(2 N-2) \times \mathrm{U}(1)_{1} \times \mathrm{U}(1)_{2},
$$

where the indices of $\mathrm{U}(1)_{1}$ and $\mathrm{U}(1)_{2}$ are only labeling the $\mathrm{U}(1)$ gauge groups and do not indicate the Chern-Simons level. The generators of these two U(1)'s are conveniently chosen as

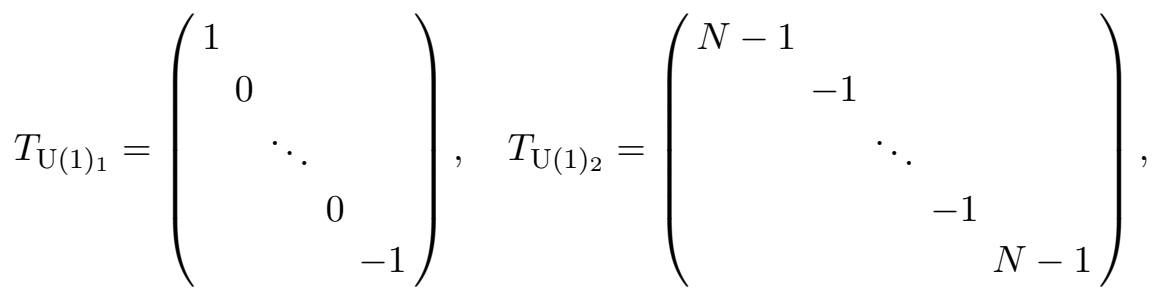

where we neglect the normalization of the generators which is not important here. Under this gauge symmetry breaking, the matter fields are decomposed as

$$
\begin{aligned}
& \square \rightarrow \square_{(0,-1)}+1_{(1, N-1)}+1_{(-1, N-1)} \\
& \square \rightarrow \square(0,1)+1_{(-1,1-N)}+1_{(1,1-N)} \\
& \square \rightarrow \square_{(0,-2)}+\square_{(1, N-2)}+\square_{(-1, N-2)}+1_{(0,2 N-2)} .
\end{aligned}
$$

Along the $Y$ direction, some of the above fields are massive and should be integrated out. Then the mixed Chern-Simons term is induced for $\mathrm{U}(1)_{1}$ and $\mathrm{U}(1)_{2}$ :

$$
k_{\text {eff }}^{\mathrm{U}(1)_{1}, \mathrm{U}(1)_{2}}=-2 N+2 .
$$

This mixed Chern-Simons term makes the Coulomb branch operator $Y$ gauge non-invariant under the $\mathrm{U}(1)_{2}$ gauge group. The $\mathrm{U}(1)_{2}$ charge of $Y$ is $2 N-2$. Then we can make this operator gauge invariant by multiplying a field in a representation $\bar{\emptyset}$ because $\bar{\square}$ decomposes under the above gauge symmetry breaking as

$$
\bar{\boxminus} \rightarrow \bar{\emptyset}_{(0,2)}+\bar{\square}_{-1,2-N}+\bar{\square}_{1,2-N}+1_{(0,2-2 N)},
$$


and we can use the last component $1_{(0,2-2 N)}$ for making the Coulomb branch operator gauge invariant. Thus we have two types of the dressed monopole operators:

$$
Y V^{N-1}, \quad Y V^{N-2} Q^{\prime 2} .
$$

Next we will replace the $\mathrm{SU}(2 N)$ dynamics with the s-confining one by assuming $g_{\mathrm{Sp}(2 N-2)}^{2} \ll g_{\mathrm{SU}(2 N)}^{2}$. The theory becomes a $3 \mathrm{~d} \mathcal{N}=2 \mathrm{Sp}(2 N)$ gauge theory with the following matter contents (table 6 ). We call this theory "magnetic".

\begin{tabular}{|c|c|c|c|c|c|c|c|}
\hline & $\mathrm{Sp}(2 N-2)$ & $\mathrm{SU}(2 N)$ & $\mathrm{SU}(2)$ & $\mathrm{U}(1)$ & $\mathrm{U}(1)$ & $\mathrm{U}(1)$ & $\mathrm{U}(1)_{R}$ \\
\hline$M:=Q \bar{Q}$ & $\square$ & $\square$ & 1 & 1 & 0 & 1 & $R_{1}+R_{3}$ \\
$M_{1}:=Q^{\prime \prime} \bar{Q}$ & 1 & $\square$ & $\square$ & 0 & 1 & 1 & $R_{2}+R_{3}$ \\
$B:=Q^{2 N-2} Q^{\prime 2}$ & 1 & 1 & 1 & $2 N-2$ & 2 & 0 & $(2 N-2) R_{1}+2 R_{2}$ \\
$\bar{B}:=\bar{Q}^{2 N}$ & 1 & 1 & 1 & 0 & 0 & $2 N$ & $2 N R_{3}$ \\
$Y_{\mathrm{SU}(2 N)}$ & 1 & 1 & 1 & $-2 N+2$ & -2 & $-2 N$ & $-(2 N-2) R_{1}-2 R_{2}-2 N R_{3}+2$ \\
$S$ & 1 & 1 & 1 & $2 N$ & 0 & 0 & $2 N R_{1}$ \\
\hline$Y_{\mathrm{Sp}(2 N-2)}^{\operatorname{mag}}$ & 1 & 1 & 1 & $-2 N$ & 0 & $-2 N$ & $-2 N R_{1}-2 N R_{3}+2$ \\
\hline
\end{tabular}

Table 6. Quantum numbers of the magnetic $\operatorname{Sp}(2 N)$ gauge theory.

In the deconfinement description with the $\mathrm{SU}(2 N) \times \mathrm{Sp}(2 N-2)$ gauge symmetry, the Sp-monopole has the fermionic zero modes coming from $Q$. Thus this Sp-monopole (instanton) should behave as the vertex for $Q$. On the other hand, in the s-confined description with the $\operatorname{Sp}(2 N-2)$ gauge symmetry, we have only the $\mathrm{SU}(2 N)$ singlets and thus the $\operatorname{Sp}(2 N)$-monopole has the fermionic zero-modes from the mesino $M:=Q \bar{Q}$. As the result, these two $\operatorname{Sp}(2 N)$-monopoles have the different types of the fermionic zero-modes although the numbers of the zero-modes are identical. Then, the naive relation $Y_{\mathrm{Sp}(2 N-2)} \sim$ $Y_{\mathrm{Sp}(2 N-2)}^{\mathrm{mag}}$ is not correct. This fact can be confirmed from the quantum numbers of these operators. More correctly the operator matching of the Coulomb branch coordinates of the $\operatorname{Sp}(2 N-2)$ gauge groups is expected to be

$$
Y_{\mathrm{Sp}(2 N-2)}=Y_{\mathrm{Sp}(2 N-2)}^{\mathrm{mag}} \bar{B}
$$

up to an unimportant normalization factor. The role of $\bar{B}$ is expected to absorb the unwanted zero-modes coming from the $\bar{Q}$ superfields. Thus the mass term for $Y_{\mathrm{Sp}(2 N-2)}$ is mapped into the Yukawa term

$$
W=S Y_{\mathrm{Sp}(2 N-2)}^{\mathrm{mag}} \bar{B}
$$

and the s-confining phase of the $\mathrm{SU}(2 N)$ gauge theory is described by

$$
W=-Y_{\mathrm{SU}(2 N)}\left(M^{2 N-2} M_{1}^{2}-B \bar{B}\right)+S Y_{\mathrm{Sp}(2 N-2)}^{\mathrm{mag}} \bar{B} .
$$

The resulting theory is again s-confining since the magnetic $\operatorname{Sp}(2 N-2)$ theory has the $2 N$ fundamentals $M$, thus we move on to the description with only the gauge singlets. The matter contents and their global charges are summarized in the following table. 


\begin{tabular}{|c|c|c|c|c|c|c|}
\hline & $\mathrm{SU}(2 N)$ & $\mathrm{SU}(2)$ & $\mathrm{U}(1)$ & $\mathrm{U}(1)$ & $\mathrm{U}(1)$ & $\mathrm{U}(1)_{R}$ \\
\hline$N:=M M$ & $\square$ & 1 & 2 & 0 & 2 & $2 R_{1}+2 R_{3}$ \\
$M_{1}$ & $\square$ & $\square$ & 0 & 1 & 1 & $R_{2}+R_{3}$ \\
$B$ & 1 & 1 & $2 N-2$ & 2 & 0 & $(2 N-2) R_{1}+2 R_{2}$ \\
$\bar{B}$ & 1 & 1 & 0 & 0 & $2 N$ & $2 N R_{3}$ \\
$Y_{\mathrm{SU}(2 N)}$ & 1 & 1 & $-2 N+2$ & -2 & $-2 N$ & $-(2 N-2) R_{1}-2 R_{2}-2 N R_{3}+2$ \\
$Y_{\mathrm{Sp}(2 N-2)}^{\mathrm{mag}}$ & 1 & 1 & $-2 N$ & 0 & $-2 N$ & $-2 N R_{1}-2 N R_{3}+2$ \\
$S$ & 1 & 1 & $2 N$ & 0 & 0 & $2 N R_{1}$ \\
\hline
\end{tabular}

Table 7. Global charges of the non-gauge theory.

The superpotential describing the low-energy dynamics is

$$
W=-Y_{\mathrm{Sp}(2 N-2)}^{\mathrm{mag}} N^{N}-Y_{\mathrm{SU}(2 N)}\left(N^{N-1} M_{1}^{2}-B \bar{B}\right)+S Y_{\mathrm{Sp}(2 N-2)}^{\mathrm{mag}} \bar{B}
$$

and the operator matching between the electric and magnetic sides is

$$
\begin{gathered}
S=V^{N}, \quad B=V^{N-1} Q^{\prime \prime 2}, \quad N=V \bar{Q}^{2}, \quad M_{1}=Q^{\prime \prime} \bar{Q}, \quad \bar{B}=\bar{Q}^{2 N} \\
Y_{\mathrm{SU}(2 N)}=Y V^{N-1}, \quad Y_{\mathrm{Sp}(2 N-2)}^{\operatorname{mag}}=Y V^{N-2} Q^{\prime \prime 2} .
\end{gathered}
$$

In this way, we can find that the $3 \mathrm{~d} \mathcal{N}=2 \mathrm{SU}(2 N)$ gauge theory with two fundamental matters, $2 N$ anti-fundamental matters and one anti-symmetric matter is an s-confining theory. This is a new s-confining theory not discussed in the literature. By introducing the mass terms for the (anti-)fundamentals, we can easily flow to the theories with the lower numbers of (anti-)fundamentals.

As the check of the above duality and the validity of the $3 \mathrm{~d}$ deconfinement method, we can derive this s-confining description from $4 \mathrm{~d}$. We know that the $4 \mathrm{~d} \mathcal{N}=1 \mathrm{SU}(2 N)$ gauge theory with one anti-symmetric matter $V, 2 N$ anti-fundamental matters $\bar{Q}$ and four fundamental matters $Q$ is s-confining $[4,32-36]$. The s-confined (magnetic) description is given by the following chiral superfields in table 8 ,

\begin{tabular}{|c|c|c|c|}
\hline & $\mathrm{SU}(2 N)$ & $\mathrm{SU}(2 N)$ & $\mathrm{SU}(4)$ \\
\hline$V$ & $\square$ & 1 & 1 \\
$\bar{Q}$ & $\square$ & $\square$ & 1 \\
$Q$ & $\square$ & 1 & $\square$ \\
\hline$M:=Q \bar{Q}$ & 1 & $\square$ & $\square$ \\
$\bar{H}:=A \bar{Q}^{2}$ & 1 & $\square$ & 1 \\
$T:=V^{N}$ & 1 & 1 & 1 \\
$H_{1}:=V^{N-1} Q^{2}$ & 1 & 1 & $\square$ \\
$H_{2}:=V^{N-2} Q^{4}$ & 1 & 1 & 1 \\
$\bar{B}:=\bar{Q}^{2 N}$ & 1 & 1 & 1 \\
\hline
\end{tabular}

Table 8. Quantum numbers of the non-gauge theory.

and the superpotential

$$
W=T M^{4} \bar{H}^{N-2}+H_{1} M^{2} \bar{H}^{N-1}+H_{2} \bar{H}^{N}+\bar{B} T H_{2}+\bar{B} H_{1}^{2},
$$


where we omitted the scaling factor for simplicity and in the table 8, we abbreviated the global U(1) symmetries. The $3 \mathrm{~d} \mathcal{N}=2$ SUSY theories and their dualities are obtained by the dimensional reduction of the $4 \mathrm{~d} \mathcal{N}=1$ dualities $[17,18]$.

First we put the electric and magnetic theories on a circle and introduce the nonperturbative superpotential coming from the Kaluza-Klein monopoles, which is absent in the s-confining magnetic description. In order to switch off the KK-monopole contribution, we need to turn on the real masses for the fundamental matters by gauging the subgroup of the $\mathrm{SU}(4)$ global symmetry, whose generator is

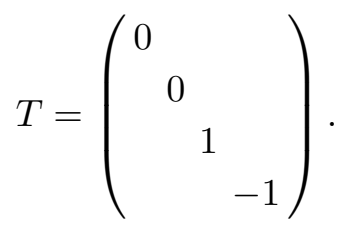

This background gauging introduces the real masses for the last two components of $Q$. If we take the low-energy limit, the superpotential induced by the KK-monopole vanishes. Then the electric theory reduces to the $3 \mathrm{~d} \mathcal{N}=2 \mathrm{SU}(2 N)$ gauge theory with one antisymmetric $V, 2 N$ anti-fundamentals and two fundamentals $Q$ without superpotentials. On the magnetic side, the following fields are only massless:

$$
\begin{gathered}
\hat{M}:=M_{a, i} \quad(a=1, \cdots, 2 N, i=1,2) \\
\bar{H}, \quad T, \quad H_{2}, \quad \bar{B} \\
h_{1}:=H_{1}^{12}, \quad h_{2}:=H_{1}^{34}
\end{gathered}
$$

The low-energy superpotential becomes

$$
W=h_{2} \hat{M}^{2} \bar{H}^{N-1}+H_{2} \bar{H}^{N}+\bar{B} T H_{2}+\bar{B} h_{1} h_{2},
$$

which is precisely the same as (3.16) if we notice the identification

$$
\begin{aligned}
& \hat{M} \sim M_{1}, \quad \bar{H} \sim N, \quad T \sim S \\
& h_{1} \sim B, \quad h_{2} \sim Y_{\mathrm{SU}(2 N)}, \quad H_{2} \sim Y_{\mathrm{Sp}(2 N-2)}^{\mathrm{mag}} .
\end{aligned}
$$

We could alternatively start with the following deconfined theory with the "s-confining $\times$ s-confining" structure (table 9 ). In this case we also have the same result except for the additional singlet to be added, then we will briefly show the result.

\begin{tabular}{|c|c|c|c|c|c|c|c|c|}
\hline & $\mathrm{SU}(2 N)$ & $\mathrm{Sp}(2 N)$ & $\mathrm{SU}(2)$ & $\mathrm{SU}(2 N)$ & $\mathrm{U}(1)$ & $\mathrm{U}(1)$ & $\mathrm{U}(1)$ & $\mathrm{U}(1)_{R}$ \\
\hline$Q$ & $\square$ & $\square$ & 1 & 1 & 1 & 0 & 0 & $R_{1}$ \\
$Q^{\prime}$ & 1 & $\square$ & $\square$ & 1 & 0 & 1 & 0 & $R_{2}$ \\
$\bar{Q}$ & $\square$ & 1 & 1 & $\square$ & 0 & 0 & 1 & $R_{3}$ \\
$S$ & 1 & 1 & 1 & 1 & $2 N$ & 2 & 0 & $2 N R_{1}+2 R_{2}$ \\
\hline$Y_{\mathrm{SU}(2 N)}$ & 1 & 1 & 1 & 1 & $-2 N$ & 0 & $-2 N$ & $-2 N R_{1}-2 N R_{3}+2$ \\
$Y_{\mathrm{Sp}(2 N)}$ & 1 & 1 & 1 & 1 & $-2 N$ & -2 & 0 & $-2 N R_{1}-2 R_{2}+2$ \\
\hline
\end{tabular}

Table 9. Quantum numbers of the deconfined theory with s-confining $\times$ s-confining structure. 
We find that both parts of the $\mathrm{SU}(2 N)$ gauge group and of the $\operatorname{Sp}(2 N)$ are in the s-confining phases. First we consider the case with $g_{\mathrm{Sp}}^{2} \gg g_{\mathrm{SU}}^{2}$ where the $\operatorname{Sp}(2 N)$ gauge theory first confines (table 10).

\begin{tabular}{|c|c|c|c|c|c|c|c|}
\hline & $\mathrm{SU}(2 N)$ & $\mathrm{SU}(2)$ & $\mathrm{SU}(2 N)$ & $\mathrm{U}(1)$ & $\mathrm{U}(1)$ & $\mathrm{U}(1)$ & $\mathrm{U}(1)_{R}$ \\
\hline$V:=Q Q$ & $\square$ & 1 & 1 & 2 & 0 & 0 & $2 R_{1}$ \\
$V^{\prime}:=Q Q^{\prime}$ & $\square$ & $\square$ & 1 & 1 & 1 & 0 & $R_{1}+R_{2}$ \\
$V^{\prime \prime}:=Q^{\prime} Q^{\prime}$ & 1 & 1 & 1 & 0 & 2 & 0 & $2 R_{2}$ \\
$\bar{Q}$ & $\square$ & 1 & $\square$ & 0 & 0 & 1 & $R_{3}$ \\
$Y_{\mathrm{Sp}(2 N)}$ & 1 & 1 & 1 & $-2 N$ & -2 & 0 & $-2 N R_{1}-2 R_{2}+2$ \\
$S$ & 1 & 1 & 1 & $2 N$ & 2 & 0 & $2 N R_{1}+2 R_{2}$ \\
\hline
\end{tabular}

Table 10. Quantum numbers of the electric theory.

We call this description "electric" and it is a $3 \mathrm{~d} \mathcal{N}=2 \mathrm{SU}(2 N)$ gauge theory with chiral (anti-)fundamental matters and an anti-symmetric matters. The superpotential is

$$
W=-Y_{\mathrm{Sp}(2 N)}\left(V^{N} V^{\prime \prime}+V^{N-1} V^{2}\right) .
$$

If we introduce the singlet field $S$ coupled to the Coulomb branch of the $\operatorname{Sp}(2 N)$ vector superfield

$$
W=S Y_{\mathrm{Sp}(2 N)},
$$

the fields $S, Y_{\mathrm{Sp}}$ are massive and integrated out, the field $V^{\prime \prime}$ is completely decouples from the other sectors. As the result, we obtain the $3 \mathrm{~d} \mathcal{N}=2 \mathrm{SU}(2 N)$ gauge theory with two fundamental matters, $2 N$ anti-fundamentals and one anti-symmetric matter without the tree-level superpotential in the infrared limit.

Next we will consider $g_{\mathrm{Sp}}^{2} \ll g_{\mathrm{SU}}^{2}$ and we can replace the dynamics of the $\mathrm{SU}(2 N)$ part with the s-confining one, in which the theory only contains the fundamentals and the gauge singlets. We call this description "magnetic theory". The quantum numbers of the magnetic theory are given in table 11 .

\begin{tabular}{|c|c|c|c|c|c|c|c|}
\hline & $\mathrm{Sp}(2 N)$ & $\mathrm{SU}(2)$ & $\mathrm{SU}(2 N)$ & $\mathrm{U}(1)$ & $\mathrm{U}(1)$ & $\mathrm{U}(1)$ & $\mathrm{U}(1)_{R}$ \\
\hline$M:=Q \bar{Q}$ & $\square$ & 1 & $\square$ & 1 & 0 & 1 & $R_{1}+R_{3}$ \\
$B:=Q^{2 N}$ & 1 & 1 & 1 & $2 N$ & 0 & 0 & $2 N R_{1}$ \\
$\bar{B}:=\bar{Q}^{2 N}$ & 1 & 1 & 1 & 0 & 0 & $2 N$ & $2 N R_{3}$ \\
$Q^{\prime}$ & $\square$ & $\square$ & 1 & 0 & 1 & 0 & $R_{2}$ \\
$Y_{\mathrm{SU}(2 N)}$ & 1 & 1 & 1 & $-2 N$ & 0 & $-2 N$ & $-2 N R_{1}-2 N R_{3}+2$ \\
$S$ & 1 & 1 & 1 & $2 N$ & 2 & 0 & $2 N R_{1}+2 R_{2}$ \\
\hline$Y_{\mathrm{Sp}(2 N)}^{\mathrm{mag}}$ & 1 & 1 & 1 & $-2 N$ & -2 & $-2 N$ & $-2 N R_{1}-2 N R_{3}-2 R_{2}+2$ \\
\hline
\end{tabular}

Table 11. Quantum numbers of the magnetic theory.

The relation between the original Coulomb branch $Y_{\mathrm{Sp}(2 N)}$ and $Y_{\mathrm{Sp}(2 N)}^{\mathrm{mag}}$ can be found from the symmetry argument as

$$
Y_{\mathrm{Sp}(2 N)}=Y_{\mathrm{Sp}(2 N)}^{\mathrm{mag}} \bar{B}
$$


so the mass term for $Y_{\mathrm{Sp}(2 N)}$ is mapped into

$$
W=S Y_{\mathrm{Sp}(2 N)}^{\mathrm{mag}} \bar{B}
$$

and the superpotential which describes the s-confining phase is

$$
W=-Y_{\mathrm{SU}(2 N)}\left(M^{2 N}-B \bar{B}\right) \text {. }
$$

The resulting theory is a $3 \mathrm{~d} \mathcal{N}=2 \operatorname{Sp}(2 N)$ gauge theory with the $2 N+2$ fundamental flavors $M, Q^{\prime}$ and the gauge singlets $S, B, \bar{B}$ and $Y_{\mathrm{SU}(2 N)}$. Notice that the magnetic $\operatorname{Sp}(2 N)$ gauge theory is again s-confining, then we have the alternative description with only the gauge singlets (table 12).

\begin{tabular}{|c|c|c|c|c|c|c|}
\hline & $\mathrm{SU}(2)$ & $\mathrm{SU}(2 N)$ & $\mathrm{U}(1)$ & $\mathrm{U}(1)$ & $\mathrm{U}(1)$ & $\mathrm{U}(1)_{R}$ \\
\hline$B:=Q^{2 N}$ & 1 & 1 & $2 N$ & 0 & 0 & $2 N R_{1}$ \\
$\bar{B}:=\bar{Q}^{2 N}$ & 1 & 1 & 0 & 0 & $2 \mathrm{~N}$ & $2 N R_{3}$ \\
$Y_{\mathrm{SU}(2 N)}$ & 1 & 1 & $-2 N$ & 0 & $-2 N$ & $-2 N R_{1}-2 N R_{3}+2$ \\
$N_{1}:=M M$ & 1 & $\square$ & 2 & 0 & 2 & $2 R_{1}+2 R_{3}$ \\
$N_{2}:=M Q^{\prime}$ & $\square$ & $\square$ & 1 & 1 & 1 & $R_{1}+R_{2}+R_{3}$ \\
$N_{3}:=Q^{\prime} Q^{\prime}$ & 1 & 1 & 0 & 2 & 0 & $2 R_{2}$ \\
$Y_{\mathrm{Sp}(2 N)}^{\mathrm{mag}}$ & 1 & 1 & $-2 N$ & -2 & $-2 N$ & $-2 N R_{1}-2 N R_{3}-2 R_{2}+2$ \\
$S$ & 1 & 1 & $2 N$ & 2 & 0 & $2 N R_{1}+2 R_{2}$ \\
\hline
\end{tabular}

Table 12. Global symmetries of the s-confining description of the magnetic theory.

Including the coupling with $S$, the superpotential is rewritten as follows:

$$
\begin{aligned}
W & =-Y_{\mathrm{SU}(2 N)}\left(M^{2 N}-B \bar{B}\right)-Y_{\mathrm{Sp}(2 N)}^{\mathrm{mag}}\left(N_{1}^{N} N_{3}+N_{1}^{N-1} N_{2}^{2}\right)+S Y_{\mathrm{Sp}(2 N)}^{\mathrm{mag}} \bar{B} \\
& \rightarrow-Y_{\mathrm{SU}(2 N)}\left(N_{1}^{N}-B \bar{B}\right)-Y_{\mathrm{Sp}(2 N)}^{\mathrm{mag}}\left(N_{1}^{N} N_{3}+N_{1}^{N-1} N_{2}^{2}\right)+S Y_{\mathrm{Sp}(2 N)}^{\operatorname{mag}} \bar{B} .
\end{aligned}
$$

This is the alternative description to the theory in table 7 . The fields $B, \bar{B}, Y_{\mathrm{SU}(2 N)}$ and $N_{1}$ in table 12 are identified with the fields $S, \bar{B}, Y_{\mathrm{Sp}(2 N-2)}^{\mathrm{mag}}$ and $N$ in table 7 . The correspondence of the remaining fields $N_{2}, N_{3}, Y_{\mathrm{Sp}(2 N)}^{\mathrm{mag}}$ and $S$ in table 12 is more subtle. The direction labeled by $S Y_{\mathrm{Sp}(2 N)}^{\mathrm{mag}}$ in table 12 corresponds to $Y_{\mathrm{SU}(2 N)} B$ in table 7 and the direction with $N_{2}^{2} Y_{\mathrm{Sp}(2 N)}^{\mathrm{mag}}$ in table 12 is matched with $Y_{\mathrm{SU}(2 N)} M_{1}^{2}$ in table $7 . N_{3}$ is identified with $V^{\prime \prime}$ in the electric theory. All the terms in (3.29), except for the third term including $N_{3}$, are translated into

$$
W \rightarrow-Y_{\mathrm{Sp}(2 N-2)}^{\mathrm{mag}}\left(N^{N}-S \bar{B}\right)-Y_{\mathrm{SU}(2 N)} N^{N-1} M_{1}^{2}+Y_{\mathrm{SU}(2 N)} B \bar{B} .
$$

This shows the precise agreement up to an introduction of $N_{3}$. However, since in the alternative description we introduce the additional singlet $V^{\prime \prime}$ and it will decouple after the use of the equation of motion, we cannot see the complete agreement between the 
superpotentials (3.16) and (3.29). In order to see the agreement of the superpotential, we can introduce the following mass term from the beginning

$$
\delta W=S^{\prime} Q^{\prime} Q^{\prime}=S^{\prime} N_{3},
$$

where $S^{\prime}$ is the new gauge singlet. This lifts the would-be decoupled direction and we recover the superpotential (3.16). Notice that the correspondence of the superpotential for the theories in table 7 and table 12 does not mean the equivalence of these two theories. We should regard the correspondence as an IR duality since the operators $N_{2}, N_{3}, Y_{\mathrm{Sp}(2 N)}^{\mathrm{mag}}$ and $S$ are mapped in a complicated way discussed above and the correspondence of the superpotential is not a simple relabeling of the fields.

\section{$3.2 \mathrm{SU}(2 N+1)$}

For the $\mathrm{SU}(2 N+1)$ gauge group, we start with the following s-cofining $\times$ s-confining theory (table 13) with an $\mathcal{N}=2$ supersymmetry. We need an additional $\operatorname{Sp}(2 N)$ fundamental matter in order to have the s-cofining $\times$ s-confining structure.

\begin{tabular}{|c|c|c|c|c|c|c|c|c|}
\hline & $\mathrm{SU}(2 N+1)$ & $\mathrm{Sp}(2 N)$ & $\mathrm{SU}(2 N+1)$ & $\mathrm{U}(1)$ & $\mathrm{U}(1)$ & $\mathrm{U}(1)$ & $\mathrm{U}(1)$ & $\mathrm{U}(1)_{R}$ \\
\hline$Q$ & $\square$ & $\square$ & 1 & 1 & 0 & 0 & 0 & $R_{1}$ \\
$Q^{\prime}$ & 1 & $\square$ & 1 & 0 & 1 & 0 & 0 & $R_{2}$ \\
$Q^{\prime \prime}$ & $\square$ & 1 & 1 & 0 & 0 & 1 & 0 & $R_{3}$ \\
$\bar{Q}$ & $\square$ & 1 & $\square$ & 0 & 0 & 0 & 1 & $R_{4}$ \\
$S$ & 1 & 1 & 1 & $2 N+1$ & 1 & 0 & 0 & $(2 N+1) R_{1}+R_{2}$ \\
\hline$Y_{\mathrm{SU}(2 N+1)}$ & 1 & 1 & 1 & $-2 N$ & 0 & -1 & $-2 N-1$ & $-2 N R_{1}-R_{3}-(2 N+1) R_{4}+2$ \\
$Y_{\mathrm{Sp}(2 N)}$ & 1 & 1 & 1 & $-2 N-1$ & -1 & 0 & 0 & $-(2 N+1) R_{1}-R_{2}+2$ \\
\hline
\end{tabular}

Table 13. Quantum numbers of the s-confining $\times$ s-confining theory for $\mathrm{SU}(2 N+1)$.

The Coulomb branch for the $\operatorname{Sp}(2 N)$ gauge group is lifted by introducing the mass term just as before.

$$
W=S Y_{\mathrm{Sp}(2 N)} .
$$

Replacing the dynamics of the $\operatorname{Sp}(2 N)$ gauge theory with the s-confining description, we have the $3 \mathrm{~d} \mathcal{N}=2 \mathrm{SU}(2 N+1)$ gauge theory with two fundamental matters, $2 N+1$ anti-fundamental matters and one anti-symmetric matter. The matter contents and their quantum numbers are summarized in the following table.

\begin{tabular}{|c|c|c|c|c|c|c|c|}
\hline & $\mathrm{SU}(2 N+1)$ & $\mathrm{SU}(2 N+1)$ & $\mathrm{U}(1)$ & $\mathrm{U}(1)$ & $\mathrm{U}(1)$ & $\mathrm{U}(1)$ & $\mathrm{U}(1)_{R}$ \\
\hline$V:=Q Q$ & $\boxminus$ & 1 & 2 & 0 & 0 & 0 & $2 R_{1}$ \\
$V^{\prime}:=Q Q^{\prime}$ & $\square$ & 1 & 1 & 1 & 0 & 0 & $R_{1}+R_{2}$ \\
$Q^{\prime \prime}$ & $\square$ & 1 & 0 & 0 & 1 & 0 & $R_{3}$ \\
$\bar{Q}$ & $\square$ & $\square$ & 0 & 0 & 0 & 1 & $R_{4}$ \\
$Y_{\mathrm{Sp}(2 N)}$ & 1 & 1 & $-2 N-1$ & -1 & 0 & 0 & $-(2 N+1) R_{1}-R_{2}+2$ \\
$S$ & 1 & 1 & $2 N+1$ & 1 & 0 & 0 & $(2 N+1) R_{1}+R_{2}$ \\
\hline
\end{tabular}

Table 14. Quantum numbers of the $\mathrm{SU}(2 N+1)$ "electric" gauge theory.

The superpotential describing the above s-confining phase is

$$
W=-Y_{\mathrm{Sp}(2 N)} V^{N} V^{\prime}+S Y_{\mathrm{Sp}(2 N)} .
$$


If we use the equation of motion of $S$, the first term in the superpotential palys no role. Then, at the low-energy limit, we have the $3 \mathrm{~d} \mathcal{N}=2 \mathrm{SU}(2 N+1)$ gauge theory with the two fundamentals, the $2 N+1$ anti-fundamentals and the anti-symmetric matter without any tree-level superpotential.

If we consider the case with $g_{\mathrm{SU}}^{2} \gg g_{\mathrm{Sp}}^{2}$, we first change the $\mathrm{SU}(2 N+1)$ dynamics to the s-confining one. The theory becomes a $3 \mathrm{~d} \mathcal{N}=2 \operatorname{Sp}(2 N)$ gauge theory with the following field contents and we call this theory "magnetic" (table 15).

\begin{tabular}{|c|c|c|c|c|c|c|c|}
\hline & $\mathrm{Sp}(2 N)$ & $\mathrm{SU}(2 N+1)$ & $\mathrm{U}(1)$ & $\mathrm{U}(1)$ & $\mathrm{U}(1)$ & $\mathrm{U}(1)$ & $\mathrm{U}(1)_{R}$ \\
\hline$M:=Q \bar{Q}$ & $\square$ & $\square$ & 1 & 0 & 0 & 1 & $R_{1}+R_{4}$ \\
$M_{1}:=Q^{\prime \prime} \bar{Q}$ & 1 & $\square$ & 0 & 0 & 1 & 1 & $R_{3}+R_{4}$ \\
$B:=Q^{2 N} Q^{\prime \prime}$ & 1 & 1 & $2 N$ & 0 & 1 & 0 & $2 N R_{1}+R_{3}$ \\
$\bar{B}:=\bar{Q}^{2 N+1}$ & 1 & 1 & 0 & 0 & 0 & $2 N+1$ & $(2 N+1) R_{4}$ \\
$Q^{\prime}$ & $\square$ & 1 & 0 & 1 & 0 & 0 & $R_{2}$ \\
$Y_{\mathrm{SU}(2 N+1)}$ & 1 & 1 & $-2 N$ & 0 & -1 & $-2 N-1$ & $-2 N R_{1}-R_{3}-(2 N+1) R_{4}+2$ \\
$S$ & 1 & 1 & $2 N+1$ & 1 & 0 & 0 & $(2 N+1) R_{1}+R_{2}$ \\
\hline$Y_{\mathrm{Sp}(2 N)}^{\mathrm{mag}}$ & 1 & 1 & $-2 N-1$ & -1 & 0 & $-2 N-1$ & $-(2 N+1) R_{1}-(2 N+1) R_{4}-R_{2}+2$ \\
\hline
\end{tabular}

Table 15. Quantum numbers of the $\operatorname{Sp}(2 N)$ "magnetic" gauge theory.

The Coulomb branch coordinates on the electric and magnetic sides are identified as

$$
Y_{\mathrm{Sp}(2 N)}=Y_{\mathrm{Sp}(2 N)}^{\mathrm{mag}} \bar{B},
$$

and the superpotential describing the s-confining phase of the part of the $\mathrm{SU}(2 N+1)$ gauge theory is

$$
W=-Y_{\mathrm{SU}(2 N+1)}\left(M^{2 N} M_{1}-B \bar{B}\right)+S Y_{\mathrm{Sp}(2 N)}^{\mathrm{mag}} \bar{B} .
$$

Notice that this theory is again s-confining, so we can go on to the non-gauge theory as follows (table 16).

\begin{tabular}{|c|c|c|c|c|c|c|}
\hline & $\mathrm{SU}(2 N+1)$ & $\mathrm{U}(1)$ & $\mathrm{U}(1)$ & $\mathrm{U}(1)$ & $\mathrm{U}(1)$ & $\mathrm{U}(1)_{R}$ \\
\hline$N:=M M$ & $\boxminus$ & 2 & 0 & 0 & 2 & $2 R_{1}+2 R_{4}$ \\
$N_{1}:=M Q^{\prime}$ & $\square$ & 1 & 1 & 0 & 1 & $R_{1}+R_{2}+R_{4}$ \\
$M_{1}$ & $\square$ & 0 & 0 & 1 & 1 & $R_{3}+R_{4}$ \\
$B$ & 1 & $2 N$ & 0 & 1 & 0 & $2 N R_{1}+R_{3}$ \\
$\bar{B}$ & 1 & 0 & 0 & 0 & $2 N+1$ & $(2 N+1) R_{4}$ \\
$Y_{\mathrm{SU}(2 N+1)}$ & 1 & $-2 N$ & 0 & -1 & $-2 N-1$ & $-2 N R_{1}-R_{3}-(2 N+1) R_{4}+2$ \\
$Y_{\mathrm{S}(2)}^{\mathrm{mag}}(2 N)$ & 1 & $-2 N-1$ & -1 & 0 & $-2 N-1$ & $-(2 N+1) R_{1}-(2 N+1) R_{4}-R_{2}+2$ \\
$S$ & 1 & $2 N+1$ & 1 & 0 & 0 & $(2 N+1) R_{1}+R_{2}$ \\
\hline
\end{tabular}

Table 16. Quantum numbers of the $\operatorname{Sp}(2 N)$ gauge theory.

The superpotential becomes

$$
W=-Y_{\mathrm{SU}(2 N+1)}\left(N^{N} M_{1}-B \bar{B}\right)-Y_{\mathrm{Sp}(2 N)}^{\mathrm{mag}} N^{N} N_{1}+S Y_{\mathrm{Sp}(2 N)}^{\mathrm{mag}} \bar{B} .
$$

The operator matching to the theory in table 14 can be easily found from the global symmetries:

$$
\begin{aligned}
N & =V \bar{Q}^{2}, & N_{1} & =V^{\prime} \bar{Q}, \\
B & =V^{N} Q^{\prime \prime}, & M_{1} & =Q^{\prime \prime} \bar{Q} \\
& =\bar{Q}^{2 N+1}, & S & =V^{N} V^{\prime} .
\end{aligned}
$$


For the case with $\mathrm{SU}(2 N)$, we can have the alternative deconfinement (table 9), which is the freedom of tuning the numbers of the $\mathrm{SU}(2 N)$ fundamentals and the Sp fundamentals. However, for $\mathrm{SU}(2 N+1)$, we only have this deconfinement since $\operatorname{Sp}(2 \tilde{N}+1)$ is not allowed. We below give the simple argument for this. Let us consider the $3 \mathrm{~d} \mathcal{N}=2$ $\mathrm{SU}(2 N+1) \times \mathrm{Sp}(2 \tilde{N})$ theory with one bi-fundamental, $F^{\prime}$ fundamentals in $\mathrm{SU}(2 N+1), F$ anti-fundamentals in $\mathrm{SU}(2 N+1)$ and $F^{\prime \prime}$ fundamentals in $\operatorname{Sp}(2 \tilde{N})$. For both of the gauge groups to be s-confining we have the following constraints

$$
\begin{aligned}
& 2 N+1+F^{\prime \prime}=2 \tilde{N}+2 \\
& F=2 \tilde{N}+F^{\prime}=2 N+1,
\end{aligned}
$$

which implies

$$
F^{\prime}=a+1, \quad F^{\prime \prime}=-a+1, \quad a:=2 N-2 \tilde{N}
$$

Since $a$ is even, only the allowed value is $a=0$, which was discussed above.

\section{3 $\mathrm{SU}(2 N)$ with an anti-symmetric flavor}

In the previous subsections, we have considered the deconfinement of the $\mathrm{SU}(N)$ gauge theory with one anti-symmetric matter and "chiral" (anti-)fundamental matters, where the anti-symmetric matter was described in terms of the s-confining theory with the Sp gauge groups. Due to the special number of the (anti-)fundamentals, the part of the $\mathrm{SU}(N)$ gauge groups is also s-confining. According to the $4 \mathrm{~d}$ case in [30], we call this structure "sconfining $\times$ s-confining". In this section, we will further study the cases with "s-confining $\times$ s-confining". We will consider the $\mathrm{SU}(2 N)$ gauge theories with matters in representations of $\square$ and $\square$.

The first example is a $3 \mathrm{~d} \mathcal{N}=2 \mathrm{SU}(4)$ gauge theory with $2(\square+\square)$ and $2 \boxminus$, where the fields $\boxminus$ and $\bar{\emptyset}$ in a SU(4) gauge group are in the same representation. This theory was first considered by [25] where it was concluded by the dimensional reduction method from $4 \mathrm{~d}$ that the theory is s-confining. The quantum numbers of the matter contents are chosen as follows (table 17) in harmony with the ones in [25]. We call this theory "electric".

\begin{tabular}{|c|c|c|c|c|c|c|c|c|}
\hline & $\mathrm{SU}(4)$ & $\mathrm{SU}(2)_{L}$ & $\mathrm{SU}(2)_{R}$ & $\mathrm{SU}(2)_{A}$ & $\mathrm{U}(1)$ & $\mathrm{U}(1)$ & $\mathrm{U}(1)$ & $\mathrm{U}(1)_{R}$ \\
\hline$A$ & $\square$ & 1 & 1 & $\square$ & 0 & 0 & -3 & 0 \\
$Q$ & $\square$ & $\square$ & 1 & 1 & 1 & 0 & 2 & $\frac{1}{3}$ \\
$\bar{Q}$ & $\square$ & 1 & $\square$ & 1 & 0 & -1 & 2 & $\frac{1}{3}$ \\
\hline
\end{tabular}

Table 17. Quantum numbers of the electric theory.

This theory has the following deconfinement description in table 18 with the $\mathrm{SU}(4) \times$ $\mathrm{SU}(2) \times \mathrm{SU}(2)$ gauge symmetry with a superpotential

$$
W=S_{1} Y_{\mathrm{SU}(2)_{1}}+S_{2} Y_{\mathrm{SU}(2)_{2}},
$$

which lifts all the $\mathrm{SU}(2) \times \mathrm{SU}(2)$ Coulomb branches. 


\begin{tabular}{|c|c|c|c|c|c|c|c|c|c|c|}
\hline & $\mathrm{SU}(4)$ & $\mathrm{SU}(2)_{1}$ & $\mathrm{SU}(2)_{2}$ & $\mathrm{SU}(2)_{L}$ & $\mathrm{SU}(2)_{R}$ & $\mathrm{U}(1)$ & $\mathrm{U}(1)$ & $\mathrm{U}(1)$ & $\mathrm{U}(1)^{\prime}$ & $\mathrm{U}(1)_{R}$ \\
\hline$x$ & $\square$ & $\square$ & 1 & 1 & 1 & 0 & 0 & $-\frac{3}{2}$ & 1 & 0 \\
$\bar{x}$ & $\square$ & 1 & $\square$ & 1 & 1 & 0 & 0 & $-\frac{3}{2}$ & -1 & 0 \\
$Q$ & $\square$ & 1 & 1 & $\square$ & 1 & 1 & 0 & 2 & 0 & $\frac{1}{3}$ \\
$\bar{Q}$ & $\square$ & 1 & 1 & 1 & $\square$ & 0 & -1 & 2 & 0 & $\frac{1}{3}$ \\
$S_{1}$ & 1 & 1 & 1 & 1 & 1 & 0 & 0 & -6 & 4 & 0 \\
$S_{2}$ & 1 & 1 & 1 & 1 & 1 & 0 & 0 & -6 & -4 & 0 \\
\hline$Y_{\mathrm{SU}(4)}$ & 1 & 1 & 1 & 1 & 1 & -2 & 2 & -2 & 0 & $\frac{2}{3}$ \\
$Y_{\mathrm{SU}(2)_{1}}$ & 1 & 1 & 1 & 1 & 1 & 0 & 0 & 6 & -4 & 2 \\
$Y_{\mathrm{SU}(2)_{2}}$ & 1 & 1 & 1 & 1 & 1 & 0 & 0 & 6 & 4 & 2 \\
\hline
\end{tabular}

Table 18. Quantum numbers of the deconfinement theory.

Note that each $\mathrm{SU}(2)_{1,2}$ gauge theory is in the s-confining phase, then we find the confined description whose matter contents are anti-symmetric matters $A_{1,2}$ and singlets $Y_{\mathrm{SU}(2)_{1,2}}$ with the $\mathrm{SU}(4)$ gauge symmetry and the following superpotentials

$$
W=-Y_{\mathrm{SU}(2)} A_{1}^{2}-Y_{\mathrm{SU}(2){ }_{2}} A_{2}^{2}
$$

where $A_{1}:=x x$ and $A_{2}:=\bar{x} \bar{x}$. These two singlets of the Coulomb branches couple to the singlets $S_{1}, S_{2}$ and become massive, then at low-energy we recover the electric theory. The electric theory has the global $\mathrm{SU}(2)_{A}$ symmetry which rotates the anti-symmetric matters, however in this deconfined theory this symmetry is not manifest. Only the U(1) subgroup of the $\mathrm{SU}(2)_{A}$ symmetry is manifest, which we denoted as $\mathrm{U}(1)^{\prime}$.

The SU(4) part of the deconfined description is also s-confining, so we can move on to the s-confined description which is a $3 \mathrm{~d} \mathcal{N}=2 \mathrm{SU}(2) \times \mathrm{SU}(2)$ gauge theory (table 19).

\begin{tabular}{|c|c|c|c|c|c|c|c|c|c|}
\hline & $\mathrm{SU}(2)_{1}$ & $\mathrm{SU}(2)_{2}$ & $\mathrm{SU}(2)_{L}$ & $\mathrm{SU}(2)_{R}$ & $\mathrm{U}(1)$ & $\mathrm{U}(1)$ & $\mathrm{U}(1)$ & $\mathrm{U}(1)^{\prime}$ & $\mathrm{U}(1)_{R}$ \\
\hline$M:=Q \bar{Q}$ & 1 & 1 & $\square$ & $\square$ & 1 & -1 & 4 & 0 & $\frac{2}{3}$ \\
$M_{1}:=Q \bar{x}$ & 1 & $\square$ & $\square$ & 1 & 1 & 0 & $\frac{1}{2}$ & -1 & $\frac{1}{3}$ \\
$M_{2}:=x \bar{Q}$ & $\square$ & 1 & 1 & $\square$ & 0 & -1 & $\frac{1}{2}$ & 1 & $\frac{1}{3}$ \\
$M_{3}:=x \bar{x}$ & $\square$ & $\square$ & 1 & 1 & 0 & 0 & -3 & 0 & 0 \\
$B:=x^{2} Q^{2}$ & 1 & 1 & 1 & 1 & 2 & 0 & 1 & 2 & $\frac{2}{3}$ \\
$\bar{B}:=\bar{x}^{2} \bar{Q}^{2}$ & 1 & 1 & 1 & 1 & 0 & -2 & 1 & -2 & $\frac{2}{3}$ \\
$Y_{\mathrm{SU}(4)}$ & 1 & 1 & 1 & 1 & -2 & 2 & -2 & 0 & $\frac{2}{3}$ \\
$S_{1}$ & 1 & 1 & 1 & 1 & 0 & 0 & -6 & 4 & 0 \\
$S_{2}$ & 1 & 1 & 1 & 1 & 0 & 0 & -6 & -4 & 0 \\
\hline$Y_{\mathrm{SU}(2)_{1}}^{\mathrm{mag}}$ & 1 & 1 & 1 & 1 & 0 & 2 & 5 & -2 & $\frac{4}{3}$ \\
$Y_{\mathrm{SU}(2)_{2}}^{\mathrm{mag}}$ & 1 & 1 & 1 & 1 & -2 & 0 & 5 & 2 & $\frac{4}{3}$ \\
\hline
\end{tabular}

Table 19. Quantum numbers of the s-confined description 1.

The s-confining superpotential is

$$
W=S_{1} Y_{\mathrm{SU}(2)_{1}}+S_{2} Y_{\mathrm{SU}(2)_{2}}-Y_{\mathrm{SU}(4)}\left(M^{2} M_{3}^{2}-M_{1}^{2} M_{2}^{2}+M M_{1} M_{2} M_{3}-B \bar{B}\right) .
$$


The relation between the Coulomb branch operators in the electric and s-confined theories is expected from the global symmetries as

$$
\begin{aligned}
& Y_{\mathrm{SU}(2)_{1}}=Y_{\mathrm{SU}(2)_{1}}^{\mathrm{mag}} \bar{B} \\
& Y_{\mathrm{SU}(2)_{2}}=Y_{\mathrm{SU}(2)_{2}}^{\mathrm{mag}} B .
\end{aligned}
$$

Both parts of the $\mathrm{SU}(2)_{1,2}$ gauge theories are again s-confining, then we have the following s-confining description (table 20).

\begin{tabular}{|c|c|c|c|c|c|c|c|c|}
\hline & $\mathrm{SU}(2)_{1}$ & $\mathrm{SU}(2)_{L}$ & $\mathrm{SU}(2)_{R}$ & $\mathrm{U}(1)$ & $\mathrm{U}(1)$ & $\mathrm{U}(1)$ & $\mathrm{U}(1)^{\prime}$ & $\mathrm{U}(1)_{R}$ \\
\hline$M$ & 1 & $\square$ & $\square$ & 1 & -1 & 4 & 0 & $\frac{2}{3}$ \\
$V:=M_{1} M_{1}$ & 1 & 1 & 1 & 2 & 0 & 1 & -2 & $\frac{2}{3}$ \\
$V_{1}:=M_{1} M_{3}$ & $\square$ & $\square$ & 1 & 1 & 0 & $-\frac{5}{2}$ & -1 & $\frac{1}{3}$ \\
$V_{2}:=M_{3} M_{3}$ & 1 & 1 & 1 & 0 & 0 & -6 & 0 & 0 \\
$M_{2}$ & $\square$ & 1 & $\square$ & 0 & -1 & $\frac{1}{2}$ & 1 & $\frac{1}{3}$ \\
$B$ & 1 & 1 & 1 & 2 & 0 & 1 & 2 & $\frac{2}{3}$ \\
$\bar{B}$ & 1 & 1 & 1 & 0 & -2 & 1 & -2 & $\frac{2}{3}$ \\
$Y_{\mathrm{SU}(4)}^{\mathrm{mag}}$ & 1 & 1 & 1 & -2 & 2 & -2 & 0 & $\frac{2}{3}$ \\
$Y_{\mathrm{SU}(2)_{2}}$ & 1 & 1 & 1 & -2 & 0 & 5 & 2 & $\frac{4}{3}$ \\
$S_{1}$ & 1 & 1 & 1 & 0 & 0 & -6 & 4 & 0 \\
$S_{2}$ & 1 & 1 & 1 & 0 & 0 & -6 & -4 & 0 \\
\hline$Y_{\mathrm{SU}(2)_{1}}^{\prime \mathrm{mag}}$ & 1 & 1 & 1 & -2 & 2 & 4 & 0 & $\frac{2}{3}$ \\
\hline
\end{tabular}

Table 20. Quantum numbers of the s-confined description 2.

The superpotential describing the second s-confining phase is

$$
\begin{aligned}
W= & S_{1} Y_{\mathrm{SU}(2)_{1}}+S_{2} Y_{\mathrm{SU}(2)_{2}}-Y_{\mathrm{SU}(4)}\left(M^{2} V_{2}-V M_{2}^{2}+M V_{1} M_{2}-B \bar{B}\right) \\
& -Y_{\mathrm{SU}(2)_{2}}^{\mathrm{mag}}\left(V V_{2}-V_{1}^{2}\right) .
\end{aligned}
$$

The relation of the Coulomb branch operators (3.43) is verified from this superpotential because if we take the equation of motion for $Y_{\mathrm{SU}(2)_{2}}^{\mathrm{mag}}$, we have $S_{2} B=V V_{2}-V_{1}^{2}$ which says $S_{2} x^{2} Q^{2}=\bar{x}^{4} x^{2} Q^{2}$. Thus $S_{2}$ is identified with the square of the anti-symmetric matters. The relation between the Coulomb branch operators of the $\mathrm{SU}(2)_{1}$ gauge group in these two s-confined descriptions is found from the symmetry argument

$$
Y_{\mathrm{SU}(2)_{1}}^{\mathrm{mag}}=Y_{\mathrm{SU}(2){ }_{1}}^{\prime \mathrm{mag}} V .
$$

The resulting $\mathrm{SU}(2)_{1}$ gauge theory is also s-confining, so the theory ends up with a non-gauge theory. The matter contents and the operator matching are summarized in table 21 . 


\begin{tabular}{|c|c|c|c|c|c|c|c|c|}
\hline & $\mathrm{SU}(2)_{L}$ & $\mathrm{SU}(2)_{R}$ & $\mathrm{U}(1)$ & $\mathrm{U}(1)$ & $\mathrm{U}(1)$ & $\mathrm{U}(1)^{\prime}$ & $\mathrm{U}(1)_{R}$ & operator matching \\
\hline$M$ & $\square$ & $\square$ & 1 & -1 & 4 & 0 & $\frac{2}{3}$ & $Q \bar{Q}$ \\
$V$ & 1 & 1 & 2 & 0 & 1 & -2 & $\frac{2}{3}$ & $A Q^{2}$ \\
$N:=V_{1}^{2}$ & 1 & 1 & 2 & 0 & -5 & -2 & $\frac{2}{3}$ & massive \\
$N_{1}:=V_{1} M_{2}$ & $\square$ & $\square$ & 1 & -1 & -2 & 0 & $\frac{2}{3}$ & $Q A^{2} \bar{Q}$ \\
$N_{2}:=M_{2} M_{2}$ & 1 & 1 & 0 & -2 & 1 & 2 & $\frac{2}{3}$ & $A \bar{Q}^{2}$ \\
$V_{2}$ & 1 & 1 & 0 & 0 & -6 & 0 & 0 & $A^{2}$ \\
$B$ & 1 & 1 & 2 & 0 & 1 & 2 & $\frac{2}{3}$ & $A Q^{2}$ \\
$\bar{B}$ & 1 & 1 & 0 & -2 & 1 & -2 & $\frac{2}{3}$ & $A \bar{Q}^{2}$ \\
$Y_{\mathrm{SU}(4)}$ & 1 & 1 & -2 & 2 & -2 & 0 & $\frac{2}{3}$ & Coulomb branch \\
$Y_{\mathrm{SU}(2)_{2}}^{\mathrm{mag}}$ & 1 & 1 & -2 & 0 & 5 & 2 & $\frac{4}{3}$ & massive \\
$Y_{\mathrm{SU}(2)_{1}}^{\prime m a g}$ & 1 & 1 & -2 & 2 & 4 & 0 & $\frac{2}{3}$ & Coulomb branch \\
$S_{1}$ & 1 & 1 & 0 & 0 & -6 & 4 & 0 & $A^{2}$ \\
$S_{2}$ & 1 & 1 & 0 & 0 & -6 & -4 & 0 & $A^{2}$ \\
\hline
\end{tabular}

Table 21. Global charges of the s-confined description 2.

The superpotential finally becomes

$$
\begin{aligned}
W= & S_{1} Y_{\mathrm{SU}(2)_{1}}^{\prime \mathrm{mag}} \bar{B} V+S_{2} Y_{\mathrm{SU}(2)_{2}}^{\mathrm{mag}} B-Y_{\mathrm{SU}(4)}\left(M^{2} V_{2}-V N_{2}+M N_{1}-B \bar{B}\right) \\
& -Y_{\mathrm{SU}(2)_{2}}^{\mathrm{mag}}\left(V V_{2}-N\right)-Y_{\mathrm{SU}(2)_{1}}^{\prime \mathrm{mag}}\left(N N_{2}-N_{1}^{2}\right) \\
= & -Y_{\mathrm{SU}(4)}\left(M^{2} V_{2}-V N_{2}+M N_{1}-B \bar{B}\right)+Y_{\mathrm{SU}(2)_{1}}^{\prime \mathrm{mag}}\left(S_{1} \bar{B} V+S_{2} B N_{2}-V V_{2} N_{2}-N_{1}^{2}\right)
\end{aligned}
$$

and the relation of the Coulomb branch operators is

$$
\begin{aligned}
Y_{\mathrm{SU}(2)_{1}} & =Y_{\mathrm{SU}(2)_{1}}^{\mathrm{mag}} \bar{B}=Y_{\mathrm{SU}(2)_{1}}^{\prime \mathrm{mag}} \bar{B} V \\
Y_{\mathrm{SU}(2)_{2}} & =Y_{\mathrm{SU}(2)_{2}}^{\mathrm{mag}} B .
\end{aligned}
$$

$N$ and $Y_{\mathrm{SU}(2)_{2}}^{\mathrm{mag}}$ are massive and integrated out. The fields listed above are completely the same as [25]. However the superpotential is slightly different. Although we expect the two superpotentials describe the same physics, but it is not clear why we obtain the different potential. One of the reasons comes from the fact that our formulation does not have the explicit $\mathrm{SU}(2)_{A}$ global symmetry.

This step of deconfining the anti-symmetric tensors is easily generalized to the $\mathrm{SU}(2 N)$ gauge theories with an anti-symmetric flavor, $\square$ and $\bar{\square}$. The deconfinement of the two antisymmetric matters can be performed by introducing the $\operatorname{Sp}(2 N-2) \times \operatorname{Sp}(2 N-2)$ gauge group. The matter contents are in table 22. In order to preserve the "s-confining $\times$ s-confining" structure, only the two fundamental flavors are included. 


\begin{tabular}{|l|c|c|c|c|c|}
\hline & $\mathrm{SU}(2 N)$ & $\mathrm{Sp}(2 N-2)_{1}$ & $\mathrm{Sp}(2 N-2)_{2}$ & $\mathrm{SU}(2)$ & $\mathrm{SU}(2)$ \\
\hline$x$ & $\square$ & $\square$ & 1 & 1 & 1 \\
$\bar{x}$ & $\square$ & 1 & $\square$ & 1 & 1 \\
$Q$ & $\square$ & 1 & 1 & $\square$ & 1 \\
$\bar{Q}$ & $\square$ & 1 & 1 & 1 & $\square$ \\
\hline
\end{tabular}

Table 22. Quantum numbers of the deconfined theory of the $\mathrm{SU}(2 N)$ with anti-symmetrics.

We omitted the global U(1) charges for simplicity of the discussion and did not listed the Coulomb branch operators in the above table. The part of the $\mathrm{SU}(2 N)$ gauge theory in the deconfined description is s-confining so we have the following theory (table 23), where we will neglect the gauge singlets throughout the rest of this section.

\begin{tabular}{|c|c|c|c|c|}
\hline & $\mathrm{Sp}(2 N-2)_{1}$ & $\mathrm{Sp}(2 N-2)_{2}$ & $\mathrm{SU}(2)$ & $\mathrm{SU}(2)$ \\
\hline$M_{1}:=Q \bar{x}$ & 1 & $\square$ & $\square$ & 1 \\
$M_{2}:=x \bar{Q}$ & $\square$ & 1 & 1 & $\square$ \\
$M_{3}:=x \bar{x}$ & $\square$ & $\square$ & 1 & 1 \\
\hline
\end{tabular}

Table 23. Quantum numbers of the s-confined description 1.

Again, since these two $\operatorname{Sp}(2 N-2)$ gauge theories are s-confining, the following confining description only with the $\operatorname{Sp}(2 N-2)_{1}$ gauge group is obtained (table 24$)$.

\begin{tabular}{|c|c|c|c|}
\hline & $\mathrm{Sp}(2 N-2)_{1}$ & $\mathrm{SU}(2)$ & $\mathrm{SU}(2)$ \\
\hline$M_{2}$ & $\square$ & 1 & $\square$ \\
$V_{1}:=M_{1} M_{3}$ & $\square$ & $\square$ & 1 \\
$V_{2}:=M_{3} M_{3}$ & $\square$ & 1 & 1 \\
\hline
\end{tabular}

Table 24. Quantum numbers of the s-confined description 2.

The $\operatorname{Sp}(2 N)$ gauge theory with one anti-symmetric and 4 fundamentals is special in the sense that it can be deconfined by the $\operatorname{Sp}(2 N-2)$ gauge theory and the deconfined description is again s-confining and that its s-confining description again has the structure with an Sp gauge theory with one anti-symmetric and 4 fundamentals. In this way we can iteratively reduce the gauge group and the ant-symmetric matters which appear in the s-confining theory can be deconfined until we end up with the $\operatorname{Sp}(2)=\mathrm{SU}(2)$ gauge theory which is also s-confining. We eventually need the $N S p$ gauge groups to completely deconfine the theory. In this process, the $N$ Coulomb branches of the Sp gauge theories are introduced and these are identified with the complicated Coulomb branches of the electric $\mathrm{SU}(2 N)$ gauge theory with $2(\square+\bar{\square})$ and $\boxminus+\bar{\square}$, which is consistent with the analysis in [25] and we find the $3 \mathrm{~d} \mathcal{N}=2 \mathrm{SU}(2 N)$ gauge theory with with $\boxminus+\boxminus$ and $2 \boxminus$ is indeed in the s-confining phase. Notice that for the deconfinement of the anti-symmetrics in the Sp gauge group, we need to subtract the "trace" part of the anti-symmetric matter but this does not spoil the above discussion.

For a $3 \mathrm{~d} \mathcal{N}=2 \mathrm{SU}(2 N+1)$ gauge theory with $2(\square+\bar{\square}), \square$ and $\dot{\square}$, we have the following deconfined description. In table 25 we only listed the gauge symmetries. 


\begin{tabular}{|c|c|c|c|}
\hline & $\mathrm{SU}(2 N+1)$ & $\mathrm{Sp}(2 N)_{1}$ & $\mathrm{Sp}(2 N)_{2}$ \\
\hline$x$ & $\square$ & $\square$ & 1 \\
$\bar{x}$ & $\square$ & 1 & $\square$ \\
$p_{1}$ & 1 & $\square$ & 1 \\
$p_{2}$ & 1 & 1 & $\square$ \\
$Q$ & $\square$ & 1 & 1 \\
$\bar{Q}$ & $\square$ & 1 & 1 \\
\hline
\end{tabular}

Table 25. Quantum numbers of the deconfinement for $\mathrm{SU}(2 N+1)$.

The Coulomb branches of the two $\operatorname{Sp}(2 N)$ 's are lifted by introducing the singlets as before. This theory again reduces to the $\operatorname{Sp}(2 N)$ gauge theory with one anti-symmetric and 4 fundamentals and with some singlets. So we find that the theory is completely s-confining and the number of the Coulomb branch coordinates is $N$. This is again consistent with [25].

\section{Gauge theories with $\mathrm{SU}(2)^{N}$ gauge group}

In the previous section, we have typically studied the $3 \mathrm{~d} \mathcal{N}=2 \mathrm{SU} \times \mathrm{Sp}$ product gauge theory, where both of the gauge dynamics are (s-)confining. Under the replacement of one of the gauge dynamics to the s-confining one, the Coulomb branch whose gauge group remains is non-trivially mapped as (3.13). This mapping can be checked from the simple consistency check and the symmetry argument but there is no rigorous proof. So it would be preferable here to generalize the "s-confining $\times$ s-confining" structure and to give the additional support on the mapping of the Coulomb branches.

In this section, we will consider the theories with the product gauge groups. We mainly focus on the product of the $\mathrm{SU}(2)$ gauge symmetries, where these $\mathrm{SU}(2)$ 's are s-confining or have the quantum modified constraint. In four-dimensions, these theories were studied for various reasons. One of the motivations was the investigation of the $\mathcal{N}=1$ Seiberg-Witten curve $[21,23,37,38]$ and the other was the derivation of the exact superpotential [22] by using the holomorphy and the "linearity principle" (integrating-in method). Here we will study the $3 \mathrm{~d}$ version and derive the $3 \mathrm{~d}$ exact superpotentials. We will find the close relation between the Seiberg-Witten curve and the exact superpotentials. The connection between the $4 \mathrm{~d}$ and $3 \mathrm{~d}$ superpotentials will give the further evidence of the mapping rule for the Coulomb branch operators.

The relation between the $4 \mathrm{~d}$ Seiberg-Witten theory and the $3 \mathrm{~d} \mathcal{N}=4$ theory is investigated in [24], in which the dimensional reduction of the curve leads to the moduli space of the $3 \mathrm{~d}$ theory. An important claim in [24] is that the variables $x, y$ describing the Seiberg-Witten curve become physical ones if we compactify the theory on $\mathbb{R}^{3} \times \mathbb{S}^{1}$. We here generalize this to the $\mathcal{N}=1$ Seiberg-Witten curve. We will thus investigate the dynamics of the product $\mathrm{SU}(2)^{N}$ gauge theory in two ways. One of them is to use a Seiberg-Witten curve and relate it to the $3 \mathrm{~d}$ superpotential. The other one is to directly study the $3 \mathrm{~d}$ theory just as we have done in the previous section. We will find that the $\mathcal{N}=1$ Seiberg-Witten curves are also dimensionally reduced and they well describe the $3 \mathrm{~d}$ moduli space. 


\subsection{Bi-fundamental and fundamental matters}

As an illustrative example, we first consider the $3 \mathrm{~d} \mathcal{N}=2 \mathrm{SU}(2) \times \mathrm{SU}(2)$ gauge theory with bi-fundamental matters and fundamental matters. The matter contents are summarized in table 26. The four-dimensional version of this theory was first investigated in [22], where the authors derived an exact superpotential using the holomorphy and the integrating-in method ("linearity principle"). We here analyze the $3 \mathrm{~d}$ version and the theory in $\mathbb{S}^{1} \times \mathbb{R}^{3}$ and find the close relation to the $4 \mathrm{~d}$ results. In the table below, we put the indices on the two $\mathrm{SU}(2)$ gauge groups for distinguishing and labeling the gauge groups. We listed the dynamical scales of the $4 \mathrm{~d}$ theory which is necessary in the $\mathbb{S}^{1} \times \mathbb{R}^{3}$ space-time. Notice that the theory in $3 \mathrm{~d}$ has the global $\mathrm{SU}(2) \times \mathrm{U}(1) \times \mathrm{U}(1) \times \mathrm{U}(1)_{R}$ symmetry, but for the $4 \mathrm{~d}$ limit the chiral anomaly reduces one combination of the $\mathrm{U}(1)$ symmetries to the spurious symmetry.

\begin{tabular}{|c|c|c|c|c|c|c|}
\hline & $\mathrm{SU}(2)_{1}$ & $\mathrm{SU}(2)_{2}$ & $\mathrm{SU}(2)$ & $\mathrm{U}(1)$ & $\mathrm{U}(1)$ & $\mathrm{U}(1)_{R}$ \\
\hline$Q$ & $\square$ & $\square$ & 1 & 1 & 0 & 0 \\
$L$ & 1 & $\square$ & $\square$ & 0 & 1 & 0 \\
\hline$Y_{\mathrm{SU}(2)_{1}}$ & 1 & 1 & 1 & -2 & 0 & 0 \\
$Y_{\mathrm{SU}(2)_{2}}$ & 1 & 1 & 1 & -2 & -2 & 2 \\
$\eta_{1}=\Lambda_{1}^{5}$ & 1 & 1 & 1 & 2 & 0 & 2 \\
$\eta_{2}=\Lambda_{2}^{4}$ & 1 & 1 & 1 & 2 & 2 & 0 \\
\hline$X:=Q Q$ & 1 & 1 & 1 & 2 & 0 & 0 \\
$Y:=L L$ & 1 & 1 & 1 & 0 & 2 & 0 \\
\hline
\end{tabular}

Table 26. Quantum numbers of the $\mathrm{SU}(2) \times \mathrm{SU}(2)$ gauge theory.

If we assume $g_{1}^{2} \ll g_{2}^{2}$ at a certain energy scale, we can replace the $\mathrm{SU}(2)_{2}$ gauge theory with two flavors to the s-confined description with the superpotential

$$
W_{\text {eff }}=-Y_{\mathrm{SU}(2)_{2}}\left(X Y-N^{2}\right),
$$

where we defined $N:=Q L$. The low-energy effective theory is an $\mathrm{SU}(2)_{1}$ gauge theory with a single flavor (two doublets). The quantum numbers are as follows (table 27).

\begin{tabular}{|c|c|c|c|c|c|}
\hline & $\mathrm{SU}(2)_{1}$ & $\mathrm{SU}(2)$ & $\mathrm{U}(1)$ & $\mathrm{U}(1)$ & $\mathrm{U}(1)_{R}$ \\
\hline$X$ & 1 & 1 & 2 & 0 & 0 \\
$Y$ & 1 & 1 & 0 & 2 & 0 \\
$N:=Q L$ & $\square$ & $\square$ & 1 & 1 & 0 \\
$Y_{\mathrm{SU}(2)_{2}}$ & 1 & 1 & -2 & -2 & 2 \\
\hline$Y_{\mathrm{SU}(2)_{1}}^{\prime}$ & 1 & 1 & -2 & -2 & 0 \\
\hline
\end{tabular}

Table 27. Quantum numbers of the $\mathrm{SU}(2)_{1}$ s-confined gauge theory.

The Coulomb branch operator $Y_{\mathrm{SU}(2)_{1}}^{\prime}$ is not identical to $Y_{\mathrm{SU}(2)_{1}}$ but we can easily identified as

$$
Y_{\mathrm{SU}(2)_{1}} \sim Y_{\mathrm{SU}(2)}^{\prime} Y,
$$

where the normalization factor is not important since we can absorb it by rescaling the chiral superfields. Since the low-energy description is the $\mathrm{SU}(2)_{1}$ gauge theory with one 
flavor, it has the quantum modified moduli space [7]. So, the theory is described by the following superpotential

$$
\begin{aligned}
W & =-Y_{\mathrm{SU}(2)_{2}}(X Y-T)+\lambda\left(T Y_{\mathrm{SU}(2)_{1}}^{\prime}-1\right) \\
& =\lambda\left(X Y Y_{\mathrm{SU}(2)_{1}}^{\prime}-1\right)
\end{aligned}
$$

where $T:=N^{2}$ and $\lambda$ is a Lagrange multiplier field. In the second line above, we integrated out $T$ and $Y_{\mathrm{SU}(2)_{2}}$. It is important to note that from this analysis we first have the flat direction labeled by $N$ but finally this direction is lifted. This is very consistent with the four dimensional analysis [22].

In order to connect the $3 \mathrm{~d}$ dynamics to the $4 \mathrm{~d}$ one, we need to put a theory on $\mathbb{S}^{1} \times \mathbb{R}^{3}$ and include the non-perturbative superpotential coming from the compactification. This superpotential is generated by the Kaluza-Klein monopole [6-8] and the form of the superpotential takes a simple form

$$
W=\eta_{1} Y_{\mathrm{SU}(2)_{1}}+\eta_{2} Y_{\mathrm{SU}(2)_{2}} .
$$

Combining this with the s-confining superpotential (4.3), integrating out the massive fields $T, Y_{\mathrm{SU}(2)_{2}}$ and $Y_{\mathrm{SU}(2)_{1}}^{\prime}$ and eliminating the Lagrange multiplier $\lambda$, we arrive at

$$
W_{\text {low-energy }}=\frac{\eta_{1} Y}{X Y-\eta_{2}} .
$$

This is precisely the same as [22] and we recover the $4 \mathrm{~d}$ dynamics. In deriving the above result, it is crucially important to use the identification (4.2) and this ahows the validity of the mapping of the Coulomb branch operators (4.2).

\subsection{Bi-fundamental matters}

Next we will consider the $3 \mathrm{~d} \mathcal{N}=2 \mathrm{SU}(2) \times \mathrm{SU}(2)$ gauge theory with only the bifundamental matters. For a single bi-fundamental, we can use the previous result by turning on the mass term to the fundamental matters. The matter contents and their quantum charges are summarized in table 28.

\begin{tabular}{|c|c|c|c|c|c|}
\hline & $\mathrm{SU}(2)_{1}$ & $\mathrm{SU}(2)_{2}$ & $\mathrm{SU}(F)$ & $\mathrm{U}(1)$ & $\mathrm{U}(1)_{R}$ \\
\hline$Q$ & $\square$ & $\square$ & $\square$ & 1 & 0 \\
\hline$Y_{1}$ & 1 & 1 & 1 & $-2 F$ & $2 F-2$ \\
$Y_{2}$ & 1 & 1 & 1 & $-2 F$ & $2 F-2$ \\
\hline$\eta_{1}:=\Lambda_{1}^{6-F}$ & 1 & 1 & 1 & $2 F$ & $4-2 F$ \\
$\eta_{2}:=\Lambda_{2}^{6-F}$ & 1 & 1 & 1 & $2 F$ & $4-2 F$ \\
\hline
\end{tabular}

Table 28. Quantum numbers of the $\mathrm{SU}(2) \times \mathrm{SU}(2)$ gauge theory with $F$ bi-fundamentals.

The four-dimensional version of this theory with $F=2$ is analyzed in [21] (for the generalization of the product gauge group, see [23, 38]). An important result of the fourdimensional analysis is that the theory shows the Coulomb phase whose rank is 1 and can be effectively described by the $\mathrm{U}(1)$ gauge theory whose gauge coupling is expressed by a $\mathcal{N}=1$ Seiberg-Witten curve. 
Here we will investigate the cases with $F=1$ and $F=2$ respectively and we will find the result for $F=2$ has intimate connection with the Seiberg-Witten curve obtained in [21]. So this is showing the $\mathcal{N}=1$ generalization of [24] where the 3 d reduction of the Seiberg-Witten curve was studied in a $4 \mathrm{~d} \mathcal{N}=2$ pure SYM with the inclusion of a softly supersymmetry breaking term.

\subsection{1 $\quad F=1$}

Suppose the $\mathrm{SU}(2)_{2}$ gauge group first becomes strong, the theory has the quantum deformed moduli space

$$
W=\lambda\left(X Y_{2}-1\right)
$$

The resulting low-energy theory has the following matter contents (table 29). $Y_{1}^{\prime}$ is a Coulomb branch operator in the low-energy $\mathrm{SU}(2)_{1}$ gauge theory.

\begin{tabular}{|c|c|c|c|}
\hline & $\mathrm{SU}(2)_{1}$ & $\mathrm{U}(1)$ & $\mathrm{U}(1)_{R}$ \\
\hline$X:=Q Q$ & 1 & 2 & 0 \\
$Y_{2}$ & 1 & -2 & 0 \\
\hline$Y_{1}^{\prime}$ & 1 & 0 & -2 \\
\hline
\end{tabular}

Table 29. Quantum numbers of the low-energy $\mathrm{SU}(2)_{1}$ gauge theory.

Since the $\mathrm{SU}(2)_{1}$ gauge theory has no flavor, there is no stable vacuum [7]:

$$
W=\lambda\left(X Y_{2}-1\right)+\frac{1}{Y_{1}^{\prime}} \text {. }
$$

This is consistent with the known result [39] since $\mathrm{SU}(2) \times \mathrm{SU}(2) \cong \mathrm{SO}(4)$ and the bi-fundamental in $\mathrm{SU}(2) \times \mathrm{SU}(2)$ is a vector matter in $\mathrm{SO}(4)$. In [39], it is shown that the $3 \mathrm{~d} \mathcal{N}=2 \mathrm{SO}(4)$ with a single flavor has no stable SUSY vacuum.

For $F=1$, we can flow from the previous subsection by the mass deformation

$$
W_{\text {def }}=m L L=m Y \text {. }
$$

Thus the effective superpotential is

$$
W=-Y_{\mathrm{SU}(2)_{2}}(X Y-T)+\lambda\left(T Y_{\mathrm{SU}(2)_{1}}^{\prime}-1\right)+m Y
$$

and this has no stable solution.

\subsection{2 $F=2$}

For $F=2$, each dynamics of the $\mathrm{SU}(2)_{1,2}$ gauge groups is in the s-confining phase. First we will change the $\mathrm{SU}(2)_{2}$ dynamics. Since the low-energy $\mathrm{SU}(2)_{1}$ gauge theory (see table 30 ) involves only the adjoint matter, we have the enhanced $\mathcal{N}=4$ supersymmetry if we omit the superpotential

$$
W=-Y_{2}\left(X_{1}^{2}+X_{2}^{2}\right),
$$

which breaks the supersymmetry from $\mathcal{N}=4$ to $\mathcal{N}=2$. 


\begin{tabular}{|c|c|c|c|c|}
\hline & $\mathrm{SU}(2)_{1}$ & $\mathrm{SU}(F=2)$ & $\mathrm{U}(1)$ & $\mathrm{U}(1)_{R}$ \\
\hline$X_{1}:=Q Q$ & adj. & 1 & 2 & 0 \\
$X_{2}:=Q Q$ & 1 & adj. & 2 & 0 \\
$Y_{2}$ & 1 & 1 & -4 & 2 \\
\hline$Y_{1}^{\prime}$ & 1 & 1 & -4 & 0 \\
\hline
\end{tabular}

Table 30. Quantum numbers of the low-energy $\mathrm{SU}(2)_{1}$ theory.

The resulting theory is the $\mathrm{SU}(2)$ gauge theory with an adjoint matter, which is regarded as the $\mathrm{SO}(3)$ gauge theory with a single fundamental (vector) matter. This theory is known to be described by the following quantum constraint [39]

$$
Y_{\mathrm{SO}(3)}^{2} N+\tilde{q}^{2}+1=0,
$$

where $N:=X_{1}^{2}$ and $Y_{\mathrm{SO}(3)}$ is a Coulomb branch operator of the $\mathrm{SO}(3)$ gauge group. $\tilde{q}$ is a dual "quark". The Seiberg dual of the $\mathrm{SO}(3)$ gauge theory with a single flavor is effectively given by the non-gauge theory, then the dual "quark" $\tilde{q}$ is a gauge singlet chiral superfield. The Coulomb branch operators between the $\mathrm{SU}(2)$ and $\mathrm{SO}(3)$ gauge groups is related as $Y_{1}^{\prime}=Y_{\mathrm{SO}(3)}^{2}$. This is very plausible since the minimal monopole charges are different in these gauge groups. Furthermore the global charges of these two operators suggest this relation. Since we are actually dealing with not the $\mathrm{SO}(3)$ but the $\mathrm{SU}(2)$ gauge group, it would be plausible to change the variables [24]

$$
Y_{\mathrm{SO}(3)}^{2} \rightarrow Y_{1}^{\prime}, \quad Y_{\mathrm{SO}(3)} \tilde{q} \rightarrow q .
$$

Under this change, the constraint becomes

$$
Y_{1}^{\prime 2} N+q^{2}+Y_{1}^{\prime}=0 .
$$

Summing up all the superpotentials and the constraint, we have the low-energy dynamics described by

$$
\begin{aligned}
W & =-Y_{2}\left(N+X_{2}^{2}\right)+\lambda\left(Y_{1}^{\prime 2} N+q^{2}+Y_{1}^{\prime}\right) \\
& \rightarrow \lambda\left(-Y_{1}^{\prime 2} X_{2}^{2}+q^{2}+Y_{1}^{\prime}\right),
\end{aligned}
$$

where in the second line we have integrated out the massive fields $Y_{2}$ and $N$. This is again consistent with the analysis of [39], where a $3 \mathrm{~d} \mathcal{N}=2 O(4)$ theory with two flavors is investigated and they claim that the Coulomb phase is described by the similar constraint. The consistency can be easily shown by the following replacement.

$$
Y_{1}^{\prime} \rightarrow Y_{O(4)}^{2}, \quad q \rightarrow \tilde{q} Y_{O(4)}
$$

Let us connect the $3 \mathrm{~d}$ dynamics with the $4 \mathrm{~d}$ one. The $4 \mathrm{~d}$ dynamics was studied in [21]. The low-energy theory is in the Coulomb phase whose rank is 1 and the gauge coupling and the singularity structure on the moduli space are captured by the following Seiberg-Witten curve

$$
y^{2}=x^{3}+x^{2}\left(-U+\Lambda_{1}^{4}+\Lambda_{2}^{4}\right)+\Lambda_{1}^{4} \Lambda_{2}^{4} x,
$$


where $U:=\operatorname{det} Q Q$. In order to dimensionally reduce the theory to $3 \mathrm{~d}$, we first notice that the $x, y$ variables are not physical ones in the $4 \mathrm{~d}$ limit but in $\mathbb{R}^{3} \times \mathbb{S}^{1}$ or in the $3 \mathrm{~d}$ limit we can treat these variables as physical fields [24]. Then we can introduce the SeibergWitten curve as the constraint between the chiral superfields $x, y$ and $U$. Notice that $U$ is a physical variable in $4 \mathrm{~d}$ and $3 \mathrm{~d}$, along which we have the massless excitation.

When we take the $3 \mathrm{~d}$ limit we should redefine the variables $x, y, U$ and absorb $\Lambda_{1}$ and $\Lambda_{2}$ since in the $3 \mathrm{~d}$ limit we must take $\Lambda_{1,2} \rightarrow 0$ and the three-dimensional gauge couplings to be fixed. According to the Seiberg-Witten prescription [24] we change the variables as

$$
\begin{aligned}
& \tilde{x}:=\left(\Lambda_{1}^{4} \Lambda_{2}^{4}\right)^{-1} x \\
& \tilde{y}:=\left(\Lambda_{1}^{4} \Lambda_{2}^{4}\right)^{-1} y \\
& v:=x-U+\Lambda_{1}^{4}+\Lambda_{2}^{4},
\end{aligned}
$$

then the Seiberg-Witten curve becomes

$$
\tilde{y}^{2}=\tilde{x}^{2} v+\tilde{x}
$$

In this expression we can safely take the $3 \mathrm{~d}$ limit and find the good agreement with the $3 \mathrm{~d}$ analysis with the identification $\tilde{y}=\tilde{q}, v=X_{2}^{2}$ and $\tilde{x}=-Y_{1}^{\prime}$.

\section{$4.3 \quad \mathrm{SU}(2) \times \mathrm{SU}(2) \times \mathrm{SU}(2)$}

We generalize the previous result on the $\mathrm{SU}(2) \times \mathrm{SU}(2)$ gauge theory with bi-fundamental matters to the theory with the $\mathrm{SU}(2) \times \mathrm{SU}(2) \times \mathrm{SU}(2)$ gauge symmetry, where the theory will have the "s-confining $\times$ s-confining $\times$ s-confining" structure. The four-dimensional version is investigated in [23], where the $\mathcal{N}=1$ Seiberg-Witten curve is derived. The matter contents and their quantum numbers are in table 31 . The theory has the global $\mathrm{U}(1)_{1} \times \mathrm{U}(1)_{2} \times \mathrm{U}(1)_{3} \times \mathrm{U}(1)_{R}$ symmetry which is anomaly-free in $3 \mathrm{~d}$.

\begin{tabular}{|c|c|c|c|c|c|c|c|}
\hline & $\mathrm{SU}(2)_{1}$ & $\mathrm{SU}(2)_{2}$ & $\mathrm{SU}(2)_{3}$ & $\mathrm{U}(1)_{1}$ & $\mathrm{U}(1)_{2}$ & $\mathrm{U}(1)_{3}$ & $\mathrm{U}(1)_{R}$ \\
\hline$Q_{1}$ & $\square$ & $\square$ & 1 & 1 & 0 & 0 & 0 \\
$Q_{2}$ & 1 & $\square$ & $\square$ & 0 & 1 & 0 & 0 \\
$Q_{3}$ & $\square$ & 1 & $\square$ & 0 & 0 & 1 & 0 \\
\hline$Y_{1}$ & 1 & 1 & 1 & -2 & 0 & -2 & 2 \\
$Y_{2}$ & 1 & 1 & 1 & -2 & -2 & 0 & 2 \\
$Y_{3}$ & 1 & 1 & 1 & 0 & -2 & -2 & 2 \\
\hline
\end{tabular}

Table 31. Quantum numbers of the triple SU(2)'s gauge theory.

Since all the gauge groups have the structure with the $\mathrm{SU}(2)$ with two flavors, the theory is reduced to the $\mathrm{SU}(2) \times \mathrm{SU}(2)$ theory by use of the s-confined description. If we first change the $\mathrm{SU}(2)_{3}$, we obtain the following theory in table 32 , where we put primes on the Coulomb branch coordinates to distinguish these operators from $Y_{1,2}$. 


\begin{tabular}{|c|c|c|c|c|c|c|}
\hline & $\mathrm{SU}(2)_{1}$ & $\mathrm{SU}(2)_{2}$ & $\mathrm{U}(1)_{1}$ & $\mathrm{U}(1)_{2}$ & $\mathrm{U}(1)_{3}$ & $\mathrm{U}(1)_{R}$ \\
\hline$Q_{1}$ & $\square$ & $\square$ & 1 & 0 & 0 & 0 \\
$N_{1}:=Q_{2} Q_{3}$ & $\square$ & $\square$ & 0 & 1 & 1 & 0 \\
$M_{2}:=Q_{2} Q_{2}$ & 1 & 1 & 0 & 2 & 0 & 0 \\
$M_{3}:=Q_{3} Q_{3}$ & 1 & 1 & 0 & 0 & 2 & 0 \\
$Y_{3}$ & 1 & 1 & 0 & -2 & -2 & 2 \\
\hline$Y_{1}^{\prime}$ & 1 & 1 & -2 & -2 & -2 & 2 \\
$Y_{2}^{\prime}$ & 1 & 1 & -2 & -2 & -2 & 2 \\
\hline
\end{tabular}

Table 32. Quantum numbers of the $\mathrm{SU}(2)_{1} \times \mathrm{SU}(2)_{2}$ gauge theory.

The superpotential is

$$
W=-Y_{3}\left(N_{1}^{2}+M_{2} M_{3}\right)
$$

and the relations between the Coulomb branch operators are

$$
Y_{1}^{\prime} M_{2}=Y_{1}, \quad Y_{2}^{\prime} M_{3}=Y_{2}
$$

The matter contents charged under the $\mathrm{SU}(2)_{1} \times \mathrm{SU}(2)_{2}$ gauge symmetry are completely the same as the previous subsection so we can reduce the $\mathrm{SU}(2) \times \mathrm{SU}(2)$ gauge symmetry to a single $\mathrm{SU}(2)$ gauge theory (table 33 ). The low-energy superpotential is

$$
W=-Y_{2}^{\prime}\left(M_{1} N_{2}+N_{3}^{2}+N_{4}^{2}\right)
$$

\begin{tabular}{|c|c|c|c|c|c|}
\hline & $\mathrm{SU}(2)_{1}$ & $\mathrm{U}(1)_{1}$ & $\mathrm{U}(1)_{2}$ & $\mathrm{U}(1)_{3}$ & $\mathrm{U}(1)_{R}$ \\
\hline$M_{1}:=Q_{1} Q_{1}$ & 1 & 2 & 0 & 0 & 0 \\
$N_{2}:=N_{1} N_{1}$ & 1 & 0 & 2 & 2 & 0 \\
$N_{3}:=\left.Q_{1} N_{1}\right|_{\text {anti-symmetric part }}$ & 1 & 1 & 1 & 1 & 0 \\
$N_{4}:=\left.Q_{1} N_{1}\right|_{\text {symmetric part }}$ & adj. & 1 & 1 & 1 & 0 \\
$Y_{3}$ & 1 & 0 & -2 & -2 & 2 \\
$Y_{2}^{\prime}$ & 1 & -2 & -2 & -2 & 2 \\
\hline$Y_{1}^{\prime \prime}$ & 1 & -2 & -2 & -2 & 0 \\
\hline
\end{tabular}

Table 33. Quantum numbers of the $\mathrm{SU}(2)_{1}$ gauge theory.

Since this theory is again the same as the previous subsection, so we have the quantum modified constraint as the low-energy description

$$
Y_{1}^{\prime \prime 2} T+\tilde{q}^{2}+Y_{1}^{\prime \prime}=0
$$


where $T:=N_{4}^{2}$. Combining all the contributions we end up with

$$
\begin{aligned}
W & =-Y_{3}\left(N_{1}^{2}+M_{2} M_{3}\right)-Y_{2}^{\prime}\left(M_{1} N_{2}+N_{3}^{2}+N_{4}^{2}\right)+\lambda\left(Y_{1}^{\prime \prime 2} T+\tilde{q}^{2}+Y_{1}^{\prime \prime}\right) \\
& =-Y_{3}\left(N_{2}+M_{2} M_{3}\right)-Y_{2}^{\prime}\left(M_{1} N_{2}+N_{3}^{2}+T\right)+\lambda\left(Y_{1}^{\prime \prime 2} T+\tilde{q}^{2}+Y_{1}^{\prime \prime}\right) .
\end{aligned}
$$

Integrateing out $Y_{3}, N_{2}, Y_{2}^{\prime}$ and $T$, we obtain the low-energy effective superpotential

$$
W=\lambda\left(Y_{1}^{\prime \prime 2}\left(M_{1} M_{2} M_{3}-N_{3}^{2}\right)+\tilde{q}^{2}+Y_{1}^{\prime \prime}\right) .
$$

This is again consistent with the Seiberg-Witten curve describing the four dimensional version of this theory

$$
y^{2}=x^{3}+x^{2}\left(\Lambda_{1}^{4} M_{2}+\Lambda_{2}^{4} M_{3}+\Lambda_{3}^{4} N_{1}-N_{1} M_{2} M_{3}+N_{3}^{2}\right)+x \Lambda_{1}^{4} \Lambda_{2}^{4} \Lambda_{3}^{4} .
$$

We can easily generalize the above analysis to the $3 \mathrm{~d} \mathcal{N}=2 \mathrm{SU}(2)^{N}$ gauge theory and obtain the low-energy effective description with the superpotential

$$
W=\lambda\left(Y^{2}\left(M_{1} \cdots M_{N}-T^{2}\right)+\tilde{q}^{2}+Y\right),
$$

where $M_{i}:=Q_{i} Q_{i}$ and $T:=Q_{1} \cdots Q_{N}$. One possible application of the $3 \mathrm{~d} \operatorname{SU}(2)^{N}$ theory is an analysis of the $3 \mathrm{~d} \mathcal{N}=2 \mathrm{SU}(4)$ gauge theory with four anti-symmetrics. This theory can be deconfined into the $3 \mathrm{~d} \mathcal{N}=2 \mathrm{SU}(4) \times \mathrm{SU}(2)^{4}$ gauge theory, in which the Coulomb branches of the $\mathrm{SU}(2)^{4}$ vector multiplets should be lifted. The SU(4) part is also in the s-confining phase and the low-energy theory reduces to the $\mathrm{SU}(2)^{4}$ gauge theory discussed above. Since the $\mathrm{SU}(4)$ gauge theory with four anti-symmetrics is equivalent to the $\mathrm{SO}(6)$ gauge theory with four vector matters, this is very consistent with the fact that the $3 \mathrm{~d}$ $\mathcal{N}=2 O\left(N_{c}\right)$ gauge theory with $N_{c}-2$ vector matters has the quantum modified constraint.

\section{$5 \mathrm{SU}(N)$ with an antisymmetric matter}

In this section we will consider the deconfinement and the Seiberg dual of the theory with an anti-symmetric matter, where the deconfined description does not have the "s-confining $\times$ s-confining" structure. Although the anti-symmetric matter is deconfined in the same way as the previous sections by using the dynamics of the $3 \mathrm{~d} \mathcal{N}=2 \mathrm{Sp}$ gauge theory, constructing the dual theory will become very complicated because in our method we are lifting up the unnecessary Coulomb moduli and the Coulomb branch operator labeling it is non-trivially mapped under the duality transformation. Then we here show two deconfinement descriptions and their duals. First, we will deconfine the anti-symmetric by lifting up the Coulomb moduli of the Sp gauge group. This is completely the same as the previous one. Next we will give the different approach, where the un-wanted Coulomb branch is just decoupled from the other sector and remaining as the massless direction.

\subsection{Lifting up the Coulomb moduli}

\subsubsection{The electric theory}

The electric theory is a $3 \mathrm{~d} \mathcal{N}=2 \mathrm{SU}(2 N)$ gauge theory with $F \square, F^{\prime} \square$ and $\square$ with no tree-level superpotential. Since there is no chiral anomaly in $3 \mathrm{~d}$, any values of $F$ and $F^{\prime}$ are allowed. The generalization to a $\mathrm{SU}(2 N+1)$ case is straightforward, so we will not discuss it. The quantum numbers of the matter contents are as follows (table 34). 


\begin{tabular}{|c|c|c|c|c|c|c|c|}
\hline & $\mathrm{SU}(2 N)$ & $\mathrm{SU}(F)$ & $\mathrm{SU}\left(F^{\prime}\right)$ & $\mathrm{U}(1)_{B}$ & $\mathrm{U}(1)_{A}$ & $\mathrm{U}(1)_{X}$ & $\mathrm{U}(1)_{R}$ \\
\hline$Q$ & $\square$ & $\square$ & 1 & 1 & 1 & 0 & 0 \\
$\tilde{Q}$ & $\square$ & 1 & $\square$ & -1 & 1 & 0 & 0 \\
$A$ & $\square$ & 1 & 1 & 0 & 0 & 2 & 0 \\
\hline
\end{tabular}

Table 34. Quantum numbers of the electric theory.

The global $\mathrm{U}(1)_{R}$ charge can be generally mixed with other $\mathrm{U}(1)$ symmetries and the genuine $\mathrm{U}(1)_{R}$ charge which is realized in the IR fixed point would not be the above combination. In this section we will consider the dual description for $F^{\prime}=F+2 N-2$ in which we can use the $3 \mathrm{~d} \mathrm{SU}\left(N_{c}\right)$ Seiberg duality developed in [17].

\subsubsection{The deconfined description}

The anti-symmetric matter can be deconfined into the $3 \mathrm{~d} \mathcal{N}=2 \mathrm{SU}(2 N) \times \operatorname{Sp}(2 N-2)$ gauge theory with the following matter contents (table 35 ):

\begin{tabular}{|c|c|c|c|c|c|c|c|c|}
\hline & $\mathrm{SU}(2 N)$ & $\mathrm{Sp}(2 N-2)$ & $\mathrm{SU}(F)$ & $\mathrm{SU}\left(F^{\prime}\right)$ & $\mathrm{U}(1)_{B}$ & $\mathrm{U}(1)_{A}$ & $\mathrm{U}(1)_{X}$ & $\mathrm{U}(1)_{R}$ \\
\hline$Q$ & $\square$ & 1 & $\square$ & 1 & 1 & 1 & 0 & 0 \\
$\tilde{Q}$ & $\square$ & 1 & 1 & $\square$ & -1 & 1 & 0 & 0 \\
$x$ & $\square$ & $\square$ & 1 & 1 & 0 & 0 & 1 & 0 \\
$S$ & 1 & 1 & 1 & 1 & 0 & 0 & $2 N$ & 0 \\
\hline$Y_{\mathrm{SU}(2 N)}$ & 1 & 1 & 1 & 1 & $2 N-2$ & $-2 F-2 N+2$ & $-2 N+2$ & $2 F-2$ \\
$Y_{\mathrm{Sp}(2 N-2)}$ & 1 & 1 & 1 & 1 & 0 & 0 & $-2 N$ & 2 \\
\hline
\end{tabular}

Table 35. Quantum numbers of the deconfined $\mathrm{SU}(2 N) \times \mathrm{Sp}(2 N-2)$ gauge theory.

The difference between the $4 \mathrm{~d}$ and $3 \mathrm{~d}$ cases is the appearance of the Coulomb branch $Y_{\mathrm{Sp}(2 N-2)}$ and the new singlet $S$ which is coupled to the unnecessary Coulomb branch direction and lifts up it. Then the deconfined theory has to include the superpotential

$$
W=S Y_{\mathrm{Sp}(2 N-2)}
$$

Applying the s-confining description of the $\operatorname{Sp}(2 N-2)$ gauge theory when $g_{\mathrm{SU}}^{2} \ll g_{\mathrm{Sp}}^{2}$, we go back to the electric theory mentioned above. The effective low-energy degrees of freedom are the mesons $A=x x$ and the Coulomb branch coordinate $Y_{\mathrm{Sp}(2 N-2)}$. However, the field $Y_{\mathrm{Sp}(2 N-2)}$ is massive due to the superpotential.

\subsubsection{The first dual description}

For $g_{\mathrm{SU}}^{2} \gg g_{\mathrm{Sp}}^{2}$, we can think of the $\mathrm{Sp}(2 N-2)$ gauge group as the flavor symmetry and we can use the $3 \mathrm{~d}$ Seiberg duality for $\mathrm{SU}\left(N_{c}\right)$ gauge group [17] if we restrict ourselves to the case with $F^{\prime}=F+2 N-2$. The dual description is given by a $3 \mathrm{~d} \mathcal{N}=2 \mathrm{U}(F-2) \times \operatorname{Sp}(2 N-2)$ gauge theory with the following matter contents (table 36 ); 


\begin{tabular}{|c|c|c|c|c|c|c|c|c|}
\hline & $\mathrm{U}(F-2)$ & $\mathrm{Sp}(2 N-2)$ & $\mathrm{SU}(F)$ & $\mathrm{SU}(F+2 N-2)$ & $\mathrm{U}(1)_{B}$ & $\mathrm{U}(1)_{A}$ & $\mathrm{U}(1)_{X}$ & $\mathrm{U}(1)_{R}$ \\
\hline$q$ & $\square$ & 1 & $\square$ & 1 & 0 & -1 & 0 & 1 \\
$\tilde{q}$ & $\square-1$ & 1 & 1 & $\square$ & 0 & -1 & 0 & 1 \\
$\tilde{x}$ & $\square_{1}$ & $\square$ & 1 & 1 & 1 & 0 & -1 & 1 \\
$b$ & $1_{-(F-2)}$ & 1 & 1 & 1 & 2 & $F$ & $2 N-2$ & $2-F$ \\
$\tilde{b}$ & $1_{+(F-2)}$ & 1 & 1 & 1 & $-2 N$ & $F+2 N-2$ & 0 & $2-F$ \\
$Y_{\mathrm{SU}(2 N)}$ & 1 & 1 & 1 & 1 & $2 N-2$ & $-2 F-2 N+2$ & $2-2 N$ & $2 F-2$ \\
$M:=Q \tilde{Q}$ & 1 & 1 & $\square$ & $\square$ & 0 & 2 & 0 & 0 \\
$M_{1}:=x \tilde{Q}$ & 1 & $\square$ & 1 & $\square$ & -1 & 1 & 1 & 0 \\
\hline$\tilde{X}_{ \pm}$ & 1 & 1 & 1 & 1 & 0 & 0 & 0 & 2 \\
$Y_{\mathrm{Sp}(2 N-2)}^{\mathrm{mag}}$ & $1_{-(F-2)}$ & 1 & 1 & 1 & $2 N$ & $-F-2 N+2$ & $-2 N$ & $F$ \\
\hline
\end{tabular}

Table 36. Quantum numbers of the first dual $\mathrm{U}(F-2) \times \operatorname{Sp}(2 N-2)$ gauge theory.

where $\tilde{X}_{ \pm}$are the Coulomb branch coordinates of the $\mathrm{U}(F-2)$ gauge group and the $Y_{\mathrm{Sp}(2 N-2)}^{\mathrm{mag}}$ is describing the $\mathrm{Sp}(2 N-2)$ Coulomb branch. For the non-zero vevs of $\tilde{X}_{ \pm}$the gauge symmetry breaks as $\mathrm{U}(F-2) \rightarrow \mathrm{U}(F-4) \times \mathrm{U}(1) \times \mathrm{U}(1)$ and the operators $\tilde{X}_{ \pm}$ create the monopoles corresponding to $\mathrm{U}(1) \times \mathrm{U}(1)$. We can not identify $Y_{\mathrm{Sp}(2 N-2)}^{\mathrm{mag}}$ with the Coulomb branch operator $Y_{\mathrm{Sp}(2 N-2)}$ of the deconfined theory. The first dual description has the superpotential

$$
W=M q \tilde{q}+M_{1} \tilde{x} \tilde{q}+Y_{\mathrm{SU}(2 N)} b \tilde{b}+\tilde{X}_{+}+\tilde{X}_{-},
$$

which is consistent with all the global symmetries listed above.

The matching of the baryonic operators between the electric and magnetic theories is

$$
\begin{aligned}
B_{l} & :=x^{l} Q^{2 N-l}=b \tilde{x}^{2 N-2-l} q^{F+l-2 N} \\
\bar{B} & :=\tilde{Q}^{2 N}=\tilde{b} \bar{q}^{F-2}
\end{aligned}
$$

and this identification removes almost all the ambiguities of the assignment of the global $\mathrm{U}(1)$ charges of the dual theory. A subtlety of the operator matching between the dual and the deconfined theories arises from the $\operatorname{Sp}(2 N-2)$ Coulomb branch operators. If we regard the $\mathrm{U}(F-2)$ gauge symmetry as the global one, the $\operatorname{Sp}(2 N-2)$ Coulomb branch operator $Y_{\mathrm{Sp}(2 N-2)}^{\mathrm{mag}}$ is negatively charged under the $\mathrm{U}(1) \subset \mathrm{U}(F-2)$ symmetry. So we can make it neutral by multiplying some fields. In this case the $\operatorname{Sp}(2 N-2)$ Coulomb branch operator is expected to be identified as

$$
Y_{\mathrm{Sp}(2 N-2)}=Y_{\mathrm{Sp}(2 N-2)}^{\mathrm{mag}} \tilde{b},
$$

which is completely consistent with all the global symmetries. Then the mass term for $Y_{\mathrm{Sp}(2 N-2)}$ becomes the Yukawa interaction

$$
W=S Y_{\mathrm{Sp}(2 N-2)}^{\mathrm{mag}} \tilde{b}
$$


Since we start with the $\mathrm{SU}(2 N)$ gauge theory with an anti-symmetric matter, then we generally have more than one-dimensional Coulomb branch. The equation of motion for $S$ says that the flat direction labeled by $Y_{\mathrm{Sp}(2 N-2)}^{\mathrm{mag}} \tilde{b}$ is lifted but the directions like

$$
Y_{\mathrm{Sp}(2 N-2)}^{\mathrm{mag}} q^{F-2}, \quad b \tilde{b}
$$

are not. The latter is lifted by the equation of motion for $Y_{\mathrm{SU}(2 N)}$. The Coulomb branch structure of the electric theory is not fully understood but this dual description suggests that $Y_{\mathrm{SU}(2 N)}$ and $Y_{\mathrm{Sp}(2 N-2)}^{\mathrm{mag}} q^{F-2}$ are identified with the Coulomb branch operators on the electric side. In the electric theory, the globally defined monopole operator $Y$ is not gauge invariant and the "dressed" operators,

$$
Y A^{N-1}, \quad Y A^{N-2} Q^{2}
$$

are expected to be gauge invariant and to parametrize the Coulomb branch because of the discussion in section 3. It is important to notice that the second one is charged under the global $\mathrm{SU}(F)$ symmetry and on the Coulomb branch the non-Abelian flavor symmetry would be broken. These operators are identified with $Y_{\mathrm{SU}(2 N)}$ and $Y_{\mathrm{Sp}(2 N-2)}^{\mathrm{mag}} q^{F-2}$ respectively from the symmetry argument. Thus the deconfinement method explains the complexity of the Coulomb branch for theories with the tensorial matters in a simpler way. However this argument does not exclude the possibility that the other types of Coulomb branch operators also remain as massless directions. The full analysis of the Coulomb branch of the electric theory will be left as the future study.

Next we can perform the Aharony dual transformation for the $\operatorname{Sp}(2 N-2)$ gauge theory [10] and the second dual theory will be given by the $3 \mathrm{~d} \mathcal{N}=2 \mathrm{U}(F-2) \times \operatorname{Sp}(2 F-4)$ gauge theory. However the theory includes the anti-symmetric matter in the $\mathrm{U}(F-2)$ gauge group and its Coulomb brach becomes very complicated. We will generally expect to need two coordinates for describing it. It is highly non-trivial how to map the operators $\tilde{X}_{ \pm}$. Since these are appearing in the superpotential, we must translate these operators in terms of the second dual theory language.

\subsubsection{The second dual description}

The second dual description is given by a $3 \mathrm{~d} \mathcal{N}=2 \mathrm{U}(F-2) \times \mathrm{Sp}(2 F-4)$ gauge theory. The matter contents are in table 37 and the superpotential is

$$
\begin{aligned}
W= & M q \tilde{q}+N_{1} \tilde{q}+Y_{\mathrm{SU}(2 N)} b \tilde{b}+\tilde{X}_{+}+\tilde{X}_{-} \\
& +N \tilde{\tilde{x}} \tilde{\tilde{x}}+N_{1} \tilde{\tilde{x}} \tilde{M}_{1}+N_{2} \tilde{M}_{1} \tilde{M}_{1}+Y_{\mathrm{Sp}(2 N-2)}^{\mathrm{mag}} Y_{\mathrm{Sp}(2 F-4)}+S Y_{\mathrm{Sp}(2 N-2)}^{\mathrm{mag}} \tilde{b}
\end{aligned}
$$

Notice that $N_{1}$ and $\tilde{q}$ are massive and integrating out these fields induces the fourth order term in the superpotential. 


\begin{tabular}{|c|c|c|c|c|c|c|c|c|}
\hline & $\mathrm{U}(F-2)$ & $\mathrm{Sp}(2 F-4)$ & $\mathrm{SU}(F)$ & $\mathrm{SU}(F+2 N-2)$ & $\mathrm{U}(1)_{B}$ & $\mathrm{U}(1)_{A}$ & $\mathrm{U}(1)_{X}$ & $\mathrm{U}(1)_{R}$ \\
\hline$q$ & $\square 1$ & 1 & $\dot{\square}$ & 1 & 0 & -1 & 0 & 1 \\
\hline$\tilde{q}$ & $\square_{-1}$ & 1 & 1 & $\dot{\square}$ & 0 & -1 & 0 & 1 \\
\hline$\tilde{\tilde{x}}$ & $\square_{-1}$ & $\square$ & 1 & 1 & -1 & 0 & 1 & 0 \\
\hline$b$ & $1_{-(F-2)}$ & 1 & 1 & 1 & 2 & $F$ & $2 N-2$ & $2-F$ \\
\hline$\tilde{b}$ & $1_{+(F-2)}$ & 1 & 1 & 1 & $-2 N$ & $F+2 N-2$ & 0 & $2-F$ \\
\hline$Y_{\mathrm{SU}(2 N)}$ & 1 & 1 & 1 & 1 & $2 N-2$ & $-2 F-2 N+2$ & $2-2 N$ & $2 F-2$ \\
\hline$M:=Q \tilde{Q}$ & 1 & 1 & $\square$ & $\square$ & 0 & 2 & 0 & 0 \\
\hline$\tilde{M}_{1}$ & 1 & $\square$ & 1 & $\dot{\square}$ & 1 & -1 & -1 & 1 \\
\hline$N:=\tilde{x} \tilde{x}$ & $\square_{2}$ & 1 & 1 & 1 & 2 & 0 & -2 & 2 \\
\hline$N_{1}:=\tilde{x} M_{1}$ & $\square_{1}$ & 1 & 1 & $\square$ & 0 & 1 & 0 & 1 \\
\hline$N_{2}:=M_{1} M_{1}$ & 1 & 1 & 1 & $\mapsto$ & -2 & 2 & 2 & 0 \\
\hline$Y_{\mathrm{Sp}(2 N-2)}^{\mathrm{mag}}$ & $1_{-(F-2)}$ & 1 & 1 & & $2 N$ & $-F-2 N+2$ & $-2 N$ & $F$ \\
\hline$\tilde{X}_{ \pm}$ & 1 & 1 & 1 & 1 & 0 & 0 & 0 & 2 \\
\hline$Y_{\mathrm{Sp}(2 F-4)}$ & $1_{+(F-2)}$ & 1 & 1 & 1 & $-2 N$ & $F+2 N-2$ & $2 N$ & $-F+2$ \\
\hline
\end{tabular}

Table 37. Quantum numbers of the second dual theory.

The problem of this description is that since the part of $\mathrm{U}(F-2)$ now includes the anti-symmetric matter $\bigoplus_{2}$, the corresponding Coulomb branch becomes highly involved. In general, we expect more than one coordinate to describe the Coulomb moduli. Here we start with the analysis of the Coulomb modui from the lower values of $F$. This analysis is inevitably required because the superpotential contains the Coulomb branch operator $\tilde{X}_{ \pm}$and these operators are the ones of the first dual theory and we need to express these operators in the second dual theory language.

For $F=3$ where the gauge group is $\mathrm{U}(1)$ and all the fields have the charges \pm 1 under this $\mathrm{U}(1)$. Notice that we cannot construct $N:=\tilde{x} \tilde{x}$ for $F=3$. This very simplifies the structure of the Coulomb branch. All the charged fermions are equally contributing to the zero-modes around the monopole background with respect to this $\mathrm{U}(1)$. The global charges of the Coulomb branch operators $\tilde{X}_{ \pm}^{\mathrm{U}(1)}$ which are related to the above monopole can be calculated by counting these fermionic zero-modes. Thus we find that the Coulomb branch operators $\tilde{X}_{ \pm}^{\mathrm{U}(1)}$ has the same global charges as $\tilde{X}_{ \pm}$. Therefore $\tilde{X}_{ \pm}$are naturally mapped to the Coulomb branch operators $\tilde{X}_{ \pm}^{\mathrm{U}(1)}$ of the second dual $\mathrm{U}(1) \times \mathrm{Sp}(2)$ gauge theory up to an irrelevant normalization factor.

For $F=4$, We have a U(2) gauge theory with the doublets, $F \square_{1},(2 F-4) \square_{-1}$, two negatively charged singlets $1_{-2}$ and two positively charged singlets $1_{+2}$, where we are not including the massive fields. Along the Coulomb branch, the $\mathrm{U}(2)$ gauge symmetry breaks to $\mathrm{U}(1)_{1} \times \mathrm{U}(1)_{2}$ and the matter fields decompose as

$$
\begin{aligned}
\square_{1} & \rightarrow 1_{(1,0)}+1_{(0,1)}, & \square_{-1} & \rightarrow 1_{(1,0)}+1_{(0,1)} \\
1_{-2} & \rightarrow 1_{(-1,-1)}, & 1_{+2} & \rightarrow 1_{(1,1)},
\end{aligned}
$$

where we renoramalized the $\mathrm{U}(1)$ 's by reorganizing the Cartan subalgebra of $\mathrm{U}(2)$ and these $\mathrm{U}(1)$ 's are defined as

$$
T_{1}=\left(\begin{array}{ll}
1 & 0 \\
0 & 0
\end{array}\right), \quad T_{2}=\left(\begin{array}{ll}
0 & 0 \\
0 & 1
\end{array}\right) .
$$

The above decomposition of the matters implies that the all the matter fields are equally 
contributing the mixed Chern-Simons terms such as

$$
k_{\text {eff }}^{\mathrm{U}(1)_{1}, \mathrm{U}(1)_{B}}=\frac{1}{2} \sum_{\text {all the fermions }} q_{\mathrm{U}(1)_{1}} q_{\mathrm{U}(1)_{B}} \operatorname{sign}(m)
$$

which determines the $\mathrm{U}(1)_{B}$ charges of the Coulomb branch operators $\tilde{X}_{ \pm}^{\mathrm{U}(2)}$. Along the Coulomb branch with $\mathrm{U}(2) \rightarrow \mathrm{U}(1) \times \mathrm{U}(1)$, we find

$$
k_{\text {eff }}^{\mathrm{U}(1)_{1}, \mathrm{U}(1)_{B}}=k_{\text {eff }}^{\mathrm{U}(1)_{1}, \mathrm{U}(1)_{A}}=k_{\text {eff }}^{\mathrm{U}(1)_{1}, \mathrm{U}(1)_{X}}=0, \quad k_{\text {eff }}^{\mathrm{U}(1)_{1}, \mathrm{U}(1)_{R}}=-2 .
$$

From the relation between the mixed Chern-Simons terms and the global U(1) charges,

$$
q^{\mathrm{U}(1)_{\text {global }}}=-k_{\mathrm{eff}}^{\mathrm{U}(1)_{1}, \mathrm{U}(1)_{\text {global }},}
$$

we find that the quantum numbers of the Coulomb branch operator $\tilde{X}_{ \pm}^{\mathrm{U}(2)}$ are identical to the ones of $\tilde{X}_{ \pm}$. We end up with the identification $\tilde{X}_{ \pm} \sim \tilde{X}_{ \pm}^{\mathrm{U}(2)}$ up to a normalization factor.

For $F=5$, we have the U(3) gauge theory with the "chiral" matters and some singlets under the $\mathrm{SU}(3) \subset \mathrm{U}(3)$. Since these matter fields have the different $\mathrm{U}(1) \sim \mathrm{U}(3) / \mathrm{SU}(3)$ charges, the Coulomb branch has the complicated quantum numbers. Under the breaking with $\mathrm{U}(3) \rightarrow \mathrm{U}(1)_{1} \times \mathrm{U}(1)_{2} \times \mathrm{U}(1)_{3}$, the matter field $\square_{2}$ decomposes into

$$
1_{(0,1,1)}+1_{(1,0,1)}+1_{(1,1,0)}
$$

This means that the effective mixed Chern-Simons term $k_{\text {eff }}^{\mathrm{U}(1)_{1}, \mathrm{U}(1)_{\text {others }}}$ has two contributions from this matter field. Noticing this subtlety we again find $\tilde{X}_{ \pm} \sim \tilde{X}_{ \pm}^{\mathrm{U}(3)}$.

For $F>5$, the $\mathrm{U}(F-2)$ theory has the anti-symmetric matter $\bigoplus_{2}$ and the Coulomb branch becomes more complicated. Then we further deconfine this anti-symmetric matter

\begin{tabular}{|c|c|c|c|c|c|c|c|c|c|}
\hline & $\mathrm{U}(F-2)$ & $\mathrm{Sp}(2 F-4)$ & $\operatorname{Sp}(F-4)$ & $\mathrm{SU}(F)$ & $\mathrm{SU}(F+2 N-2)$ & $\mathrm{U}(1)_{B}$ & $\mathrm{U}(1)_{A}$ & $\mathrm{U}(1)_{X}$ & $\mathrm{U}(1)_{R}$ \\
\hline$q$ & $\square_{1}$ & 1 & 1 & $\dot{\square}$ & 1 & 0 & -1 & 0 & 1 \\
\hline$\tilde{\tilde{x}}$ & $\bar{\square}_{-1}$ & $\square$ & 1 & 1 & 1 & -1 & 0 & 1 & 0 \\
\hline$b$ & $1_{-(F-2)}$ & 1 & 1 & 1 & 1 & 2 & $F$ & $2 N-2$ & $2-F$ \\
\hline$\tilde{b}$ & $1_{+(F-2)}$ & 1 & 1 & 1 & 1 & $-2 N$ & $F+2 N-2$ & 0 & $2-F$ \\
\hline$Y_{\mathrm{SU}(2 N)}$ & 1 & 1 & 1 & 1 & 1 & $2 N-2$ & $-2 F-2 N+2$ & $2-2 N$ & $2 F-2$ \\
\hline$M:=Q \tilde{Q}$ & 1 & 1 & 1 & $\square$ & $\square$ & 0 & 2 & 0 & 0 \\
\hline$\tilde{M}_{1}$ & 1 & $\square$ & 1 & 1 & $\dot{\square}$ & 1 & -1 & -1 & 1 \\
\hline$y$ & $\square 1$ & 1 & $\square$ & 1 & 1 & 1 & 0 & -1 & 1 \\
\hline$N_{2}:=M_{1} M_{1}$ & 1 & 1 & 1 & 1 & 曰 & -2 & 2 & 2 & 0 \\
\hline$Y_{\mathrm{Sp}(2 N-2)}^{\mathrm{mag}}$ & $1_{-(F-2)}$ & 1 & 1 & 1 & 1 & $2 N$ & $-F-2 N+2$ & $-2 N$ & $F$ \\
\hline$S^{\prime}$ & $1_{+(F-2)}$ & 1 & 1 & 1 & 1 & $F-2$ & 0 & $2-F$ & $F-2$ \\
\hline$\tilde{X}_{ \pm}$ & 1 & 1 & 1 & 1 & 1 & 0 & 0 & 0 & 2 \\
\hline$Y_{\mathrm{Sp}(2 F-4)}$ & 1 & 1 & 1 & 1 & 1 & $-2 N$ & $F+2 N-2$ & $2 N$ & $-F+2$ \\
\hline$Y_{\mathrm{Sp}(F-4)}$ & $1_{-(F-2)}$ & 1 & 1 & 1 & 1 & $2-F$ & 0 & $F-2$ & $4-F$ \\
\hline$X_{ \pm}^{\mathrm{dec}}$ & 1 & 1 & 1 & 1 & 1 & 0 & 0 & 0 & 2 \\
\hline
\end{tabular}
by introducing the $\operatorname{Sp}(F-4)$ gauge theory, where we are assuming $F$ is even, but it is easily generalized to the odd $F$ case which we will not discuss here. The deconfined theory has the following matter contents and their global charges (table 38).

Table 38. Quantum numbers of the second deconfined theory. 
The anti-symmetric matter $N$ is deconfined into $y$ and we introduce a singlet in order to lift up the Coulomb moduli of $\operatorname{Sp}(F-4)$. The important point is that the Coulomb moduli $Y_{\mathrm{Sp}(F-4)}$ is charged under the $\mathrm{U}(1)$ gauge group which is the abelian part of $\mathrm{U}(F-2)$. Then $S^{\prime}$ is also charged under the $\mathrm{U}(F-2)$ and contributes as the zero-mode around the monopole background. The superpotential now includes $\delta W=S^{\prime} Y_{\mathrm{Sp}(F-4)}$ and this term is very non-trivial from the UV field theory point of view. In order to avoid this difficulty, we will discus the different approach of deconfining the two-index matters in the next subsection. Summing up the all the contributions to the superpotential, we have

$$
\begin{aligned}
W= & \left.-M q \tilde{\tilde{x}} \tilde{M}_{1}+Y_{\mathrm{SU}(2 N)}\right) \tilde{b}+\tilde{X}_{+}+\tilde{X}_{-} \\
& +\left(\text {yy) } \tilde{\tilde{x}} \tilde{\tilde{x}}+N_{2} \tilde{M}_{1} \tilde{M}_{1}+Y_{\mathrm{Sp}(2 N-2)}^{\mathrm{mag}} Y_{\mathrm{Sp}(2 F-4)}+S Y_{\mathrm{Sp}(2 N-2)}^{\mathrm{mag}} \tilde{b}+S^{\prime} Y_{\mathrm{Sp}(F-4)} .\right.
\end{aligned}
$$

We can easily find that $\tilde{X}_{ \pm}$are mapped to the Coulomb branch operators $X_{ \pm}^{\text {dec }}$ of the second deconfined $\mathrm{U}(F-2) \times \mathrm{Sp}(2 F-4) \times \mathrm{Sp}(F-4)$ gauge theory.

In this way, we can understand the Coulomb brach dynamics of the theory with the two-index matter from the quantum dynamics of the theory with only the fundamental matters. This is the advantage of the deconfinement.

\subsection{Decoupling the Coulomb moduli}

Finally we show the different approach, where the unnecessary Coulomb branch is not lifted but remains as the flat direction and it will be completely decoupled from the other sector. Let us again consider the $3 \mathrm{~d} \mathcal{N}=2 \mathrm{SU}(N)$ gauge theory with a single anti-symmetric, $F$ fundamentals and $F^{\prime}=F+2 N-2$ anti-fundamentals without a tree-level superpotential. The theory is deconfined into the following theory in table 39 without the introduction of the singlet lifting up the Coulomb branch. We need instead include the additional matter fields and the superpotential

$$
W=x p_{1} p_{2}+p_{1} p_{1} p_{3}
$$

\begin{tabular}{|c|c|c|c|c|c|c|c|c|c|}
\hline & $\mathrm{SU}(N)$ & $\mathrm{Sp}(\mathrm{N}+\mathrm{K}-2)$ & $\mathrm{SU}(F)$ & $\mathrm{SU}\left(F^{\prime}\right)$ & $\mathrm{SU}(\mathrm{K})$ & $\mathrm{U}(1)_{B}$ & $\mathrm{U}(1)_{A}$ & $\mathrm{U}(1)_{X}$ & $\mathrm{U}(1)_{R}$ \\
\hline$Q$ & $\square$ & 1 & $\square$ & 1 & 1 & 1 & 1 & 0 & 0 \\
$\tilde{Q}$ & $\square$ & 1 & 1 & $\square$ & 1 & -1 & 1 & 0 & 0 \\
$x$ & $\square$ & $\square$ & 1 & 1 & 1 & 0 & 0 & $\frac{1}{2}$ & 0 \\
$p_{1}$ & 1 & $\square$ & 1 & 1 & $\square$ & $B$ & $A$ & $X$ & $R$ \\
$p_{2}$ & $\square$ & 1 & 1 & 1 & $\square$ & $-B$ & $-A$ & $-\frac{1}{2}-X$ & $2-R$ \\
$p_{3}$ & 1 & 1 & 1 & 1 & $\square$ & $-2 B$ & $-2 A$ & $-2 X$ & $2-2 R$ \\
\hline$Y_{\mathrm{Sp}}$ & 1 & 1 & 1 & 1 & 1 & $-B K$ & $-A K$ & $-\frac{1}{2} N-X K$ & $-R K+2$ \\
\hline
\end{tabular}

Table 39. Quantum numbers of the deconfined $\mathrm{SU}(N) \times \operatorname{Sp}(N+K-2)$ gauge theory.

Notice that in this theory the additional matter fields are included unlike the previous subsection and this is very similar to the $4 \mathrm{~d}$ deconfinement [5]. Here we leave the ambiguities of the $\mathrm{U}(1)$ charges. For even $N$, we should take even $K$. For odd $N, K$ should be 
odd and we can take $K=1$. In that case, we don't need a $p_{3}$ field because the meson $p_{1} p_{1}$ cannot be constructed. Then the superpotential is simply $W=x p_{1} p_{2}$.

If we assume that the $\mathrm{Sp}(N+K-2)$ theory first confines, then the low-energy theory is described by the mesons $A=x x, A_{1}:=x p_{1}, A_{2}:=p_{1} p_{1}$ and the Coulomb branch operator $Y_{\mathrm{Sp}}$ with the effective superpotential

$$
W=A_{1} p_{2}+A_{2} p_{3}-Y_{\mathrm{Sp}} \operatorname{Pf}\left(\begin{array}{cc}
A & A_{1} \\
-A_{1} & A_{2}
\end{array}\right) .
$$

The mesons $A_{1}$ and $A_{2}$ are massive due to the tree-level superpotential, then the effective superpotential is vanishing and plays no role. The important point is that the Coulomb branch operator $Y_{\mathrm{Sp}}$ is completely decoupled from the other sector of the theory. Thus we recover the electric $\mathrm{SU}(N)$ gauge theory with $F$ fundamentals, $F^{\prime}$ anti-fundamentals and an anti-symmetric without a superpotential.

For $F^{\prime}=F+N-2$, the $\mathrm{SU}(N)$ theory has the dual description [17] with the (nonspecial) unitary gauge group. The dual is given by a $\mathrm{U}(F+K-2) \times \mathrm{Sp}(N+K-2)$ gauge theory. The superpotential is

$$
W=M_{3} p_{1}+p_{1} p_{1} p_{3}+M q \tilde{q}+M_{1} q \tilde{p}_{2}+M_{2} \tilde{x} \tilde{q}+M_{3} \tilde{x} \tilde{p}_{2}+Y_{\mathrm{SU}} b \tilde{b}+\tilde{X}_{+}+\tilde{X}_{-},
$$

\begin{tabular}{|c|c|c|c|c|c|c|c|c|c|}
\hline & $\mathrm{U}(F+K-2)$ & $\mathrm{Sp}(N+K-2)$ & $\mathrm{SU}(F)$ & $\mathrm{SU}(F+N-2)$ & $\mid \mathrm{SU}(\mathrm{K})$ & $\mathrm{U}(1)_{B}$ & $\mathrm{U}(1)_{A}$ & $\mathrm{U}(1)_{X}$ & $\mathrm{U}(1)_{R}$ \\
\hline$q$ & $\square_{1}$ & 1 & 口 & 1 & 1 & $B-1$ & $A-1$ & $X+\frac{1}{2}$ & $R$ \\
\hline$\tilde{q}$ & $\bar{D}_{-1}$ & 1 & 1 & 口 & 1 & $1-B$ & $-A-1$ & $-X-\frac{1}{2}$ & $2-R$ \\
\hline$\tilde{x}$ & $\square_{1}$ & 口 & 1 & 1 & 1 & $B$ & $A$ & $x$ & $R$ \\
\hline$p_{1}$ & 1 & 口 & 1 & 1 & 口 & $B$ & $A$ & $x$ & $R$ \\
\hline$\tilde{p}_{2}$ & $\bar{\square}_{-1}$ & 1 & 1 & 1 & $\square$ & 0 & 0 & 0 & 0 \\
\hline$p_{3}$ & 1 & 1 & 1 & 1 & $\overline{\mathrm{\theta}}$ & $-2 B$ & $-2 A$ & $-2 X$ & $2-2 R$ \\
\hline$b$ & $1_{-(F+K-2)}$ & 1 & 1 & 1 & 1 & $-B(F+K-2)+F$ & $F-A(F+K-2)$ & $-X(F+K-2)-\frac{1}{2}(F-N)$ & $-R(F+K-2)$ \\
\hline$\tilde{b}$ & $1_{F+K-2}$ & 1 & 1 & 1 & 1 & $B(F-2)-N-F+2$ & $A(F-2)+N+F-2$ & $X(F-2)+\frac{1}{2} F-1$ & $R(F-2)-2 F+4$ \\
\hline$Y_{\mathrm{SU}}$ & 1 & 1 & 1 & 1 & 1 & $B K+N-2$ & $A K-2 F-N+2$ & $X K-\frac{1}{2} N+1$ & $R K+2 F-2$ \\
\hline$M=Q \tilde{Q}$ & 1 & 1 & $\square$ & 口 & 1 & 0 & 2 & 0 & 0 \\
\hline$M_{1}=Q p_{2}$ & 1 & 1 & $\square$ & 1 & 口 & $1-B$ & $1-A$ & $-\frac{1}{2}-X$ & $2-R$ \\
\hline$M_{2}=x \tilde{Q}$ & 1 & 口 & 1 & 口 & 1 & -1 & 1 & $\frac{1}{2}$ & 0 \\
\hline$M_{3}=x p_{2}$ & 1 & 口 & 1 & 1 & 口 & $-B$ & $-A$ & $-{ }^{2}$ & $2-R$ \\
\hline$Y_{\mathrm{Sp}}^{\operatorname{mag}}$ & $1_{-(F+K-2)}$ & 1 & 1 & 1 & 1 & $-B K-B F+2 B+F+N-2$ & $-A K-A F+2 A-F-N+2$ & $-X F-X K+2 X-\frac{1}{2} F-\frac{1}{2} N+1$ & $-K R-F R+2 R+2 F-2$ \\
\hline
\end{tabular}

and the matter contents are summarized in table 40 .

Table 40. Quantum numbers of the first dual $\mathrm{U}(F+K-2) \times \mathrm{Sp}(N+K-2)$ gauge theory.

The advantage of this method is that the un-needed Coulomb branch is automatically decoupled then we don't worry about how to map these Coulomb branch operators and we can apply the conventional Seiberg or Aharony dualities without worrying about the UV realization of the terms such as $S Y_{\mathrm{Sp}}$. However, it is again difficult to see what degrees of freedom are decoupled from the dual theory. It would be interesting to see what degrees of freedom are actually decoupled by using the index calculations or the method presented in [40]. This will be left as the future work. In this first dual description, we expect that the Coulomb branch operator of the deconfined description $Y_{\mathrm{Sp}}$ relates to the Coulomb branch operator of the $\operatorname{Sp}(N+K-2)$ gauge group in a following way:

$$
Y_{\mathrm{Sp}}=Y_{\mathrm{Sp}}^{\operatorname{mag} \tilde{b}} .
$$

This implies that the combination $Y_{\mathrm{Sp}}^{\mathrm{mag}} \tilde{b}$ is completely decouples from the other moduli. This is highly non-trivial statement and it should be checked. 
The superpotential gives the mass terms between the fields $M_{3}$ and $p_{1}$, then we can integrate out them. Then the theory reduces to

$$
W=\left(\tilde{x} \tilde{p}_{2}\right)\left(\tilde{x} \tilde{p}_{2}\right) p_{3}+M q \tilde{q}+M_{1} q \tilde{p}_{2}+M_{2} \tilde{x} \tilde{q}+Y_{\mathrm{SU}} b \tilde{b}+\tilde{X}_{+}+\tilde{X}_{-}
$$

with the following matter contents (table 41).

\begin{tabular}{|c|c|c|c|c|c|c|c|c|c|}
\hline & $\mathrm{U}(F+K-2)$ & $\mid \mathrm{Sp}(N+K-2)$ & $\mathrm{SU}(F)$ & $\mathrm{SU}(F+N-2)$ & \begin{tabular}{|l|}
$\mathrm{SU}(\mathrm{K})$ \\
\end{tabular} & $\mathrm{U}(1)_{B}$ & $\mathrm{U}(1)_{A}$ & $\mathrm{U}(1)_{X}$ & $\mathrm{U}(1)_{R}$ \\
\hline$q$ & $\square_{1}$ & 1 & प & 1 & 1 & $B-1$ & $A-1$ & $X+\frac{1}{2}$ & $R$ \\
\hline$\tilde{q}$ & $\mathrm{a}_{-1}$ & 1 & 1 & 官 & 1 & $1-B$ & $-A-1$ & $-X-\frac{1}{2}$ & $2-R$ \\
\hline$\tilde{x}$ & $\square_{1}$ & 口 & 1 & 1 & 1 & $B$ & $A$ & $x$ & $R$ \\
\hline$\tilde{p}_{2}$ & $\tilde{\mathrm{a}}_{-1}$ & 1 & 1 & 1 & $\square$ & 0 & 0 & 0 & 0 \\
\hline$p_{3}$ & 1 & 1 & 1 & 1 & E & $-2 B$ & $-2 A$ & $-2 X$ & $2-2 R$ \\
\hline$b$ & $1_{-(F+K-2)}$ & 1 & 1 & 1 & 1 & $-B(F+K-2)+F$ & $F-A(F+K-2)$ & $-X(F+K-2)-\frac{1}{2}(F-N)$ & $-R(F+K-2)$ \\
\hline$\tilde{b}$ & $1_{F+K-2}$ & 1 & 1 & 1 & 1 & $B(F-2)-N-F+2$ & $A(F-2)+N+F-2$ & $X(F-2)+\frac{1}{2} F-1$ & $R(F-2)-2 F+4$ \\
\hline$Y_{\mathrm{SU}}$ & 1 & 1 & 1 & 1 & 1 & $B K+N-2$ & $A K-2 F-N+2$ & $X K-\frac{1}{2} N+1$ & $R K+2 F-2$ \\
\hline$s$ & 1 & 1 & 1 & 1 & 1 & $B K$ & $A K$ & $\frac{1}{2} N+X K$ & $R K$ \\
\hline$M=Q \tilde{Q}$ & 1 & 1 & $\square$ & ㅁ & 1 & 0 & 2 & 0 & 0 \\
\hline$M_{1}=Q p_{2}$ & 1 & 1 & $\square$ & 1 & $\overline{0}$ & $1-B$ & $1-A$ & $-\frac{1}{2}-X$ & $2-R$ \\
\hline$M_{2}=x \tilde{Q}$ & 1 & $\square$ & 1 & $\square$ & 1 & -1 & 1 & $\frac{1}{2}$ & 0 \\
\hline$Y_{s p}^{\operatorname{mag}}$ & $1_{-(F+K-2)}$ & 1 & 1 & 1 & 1 & $-B K-B F+2 B+F+N-2$ & $-A K-A F+2 A-F-N+2$ & $-X F-X K+2 X-\frac{1}{2} F-\frac{1}{2} N+1$ & $-K R-F R+2 R+2 F-2$ \\
\hline
\end{tabular}

Table 41. Quantum numbers of the low-energy first dual theory.

Finally one may expect that the method presented in this subsection can be applied to the theories discussed in section 3. We can generally use this method but the resulting deconfined theory has no "s-confining $\times$ s-confining" structure and we cannot reproduce the s-confining superpotential. At this stage we have no natural answer to explain the discrepancy and this will be left as the future work.

\section{Summary and discussion}

In this paper we developed a deconfinement technique in the $3 \mathrm{~d} \mathcal{N}=2$ supersymmetric gauge teories and investigated the dynamics of the product gauge group with special attention to their s-confining phases. This method is quite general and would be applicable to any two-index matters ${ }^{3}$ and general product gauge groups. We here concentrated on the anti-symmetric matters which were deconfined by the s-confining phase of the $3 \mathrm{~d} \mathcal{N}=2$ $\operatorname{Sp}(2 N)$ gauge theory. The mesonic operators in the s-confining phase describe the Higgs branch and they are identified with the two-index matters we want. The difference between the $3 \mathrm{~d}$ and $4 \mathrm{~d}$ deconfinement methods is the presence of the Coulomb branch coordinates of the s-confining gauge theories. The unnecessary flat directions are lifted by introducing the additional singlets and by coupling them to the Coulomb branch coordinates as the mass terms. In the "s-confining $\times$ s-confining" theories, these additional singlets are

\footnotetext{
${ }^{3}$ For the symmetric matters, we will be able to use the s-confining phase of the $3 \mathrm{~d} \mathcal{N}=2 O(N)$ gauge theory with $F=N-1$ vector matters, where the s-confining description includes the symmetric meson, the Coulomb branch operator and the dual gauge-singlet quarks. For the purpose of the deconfinemnt of the symmetric matters, we need lift up the Coulomb branch and the dual quarks by introducing the additional gauge singlets. This seems different from the $4 \mathrm{~d}$ deconfinement of the symmetric matter. Since the lowenergy dynamics of the $4 \mathrm{~d} \mathcal{N}=1 \mathrm{SO}(N)$ gauge theory with $N-4$ vector matters has two inequivalent branches where one of them is confining and the other has no stable vacuum [41]. Thus in $4 \mathrm{~d}$, we have to omit the second branch and we have to choose the confining phase. In this sense the deconfinement of the symmetric matter is not well investigated in $4 \mathrm{~d}$. In $3 \mathrm{~d}$, we will not have such a problem. For the adjoint matters, we can use the s-confining phase of the $3 \mathrm{~d} \mathcal{N}=2 \mathrm{SU}(N)$ or $\mathrm{U}(N)$ gauge theories.
} 
identified with the gauge singlets which consist of the two-index matters. For the theory with an anti-symmetric flavor, we have found the same massless direction as [25] but the form of the superpotential is slightly different. We have no satisfying reason to explain this discrepancy but we expect these two potentials describe the same physics. This will be left as the future work. For the analysis of the $3 \mathrm{~d} \mathcal{N}=2 \mathrm{SU}(2)^{N}$ product gauge theory, we derived the exact superpotential which describes the low-energy $3 \mathrm{~d}$ dynamics and this is highly consistent with the exact superpotential in $4 \mathrm{~d}$ and with the Seiberg-Witten curve.

The deconfined technique is usually not so useful because the dynamics of either $\mathrm{SU}(N)$ or $\operatorname{Sp}\left(2 N^{\prime}\right)$ gauge group is alway strong in the infrared region and we do not have the weakly coupled description. This situation is the same for the $3 \mathrm{~d}$ deconfinement and what is even worse is that, in $3 \mathrm{~d}$, all the gauge theories become strong even if the $\mathrm{U}(1)$ gauge theories are considered. However, while the $3 \mathrm{~d}$ theories with two-index matters generally have the very complicated Coulomb moduli, the deconfined description have more tractable Coulomb branch since there are only the (bi-)fundamental matters. This is one of the advantages of the deconfinement in $3 \mathrm{~d}$. One direction of the Coulomb branch in the $\mathrm{SU}(2 N)$ theory with an anti-symmetric matter is related to the Coulomb branch of the $\mathrm{Sp}(2 N-2)$ gauge group in the deconfined $\mathrm{SU}(2 N) \times \mathrm{Sp}(2 N-2)$ theory. If we go on to the dual description of the $\mathrm{SU}(2 N) \times \mathrm{Sp}(2 N-2)$ gauge theory, we can further find the Coulomb branch operator labeling the remaining part of the Coulomb branch of the electric theory. However the mapping of the full Coulomb branch operators between the electric and deconfined description is non-trivial, which will be left as a future study.

Furthermore, we have shown that the $3 \mathrm{~d}$ deconfinement with "s-confining $\times$ s-confining" structure gives the completely s-confining description with no gauge group for the $\mathrm{SU}(N)$ gauge theory with anti-symmetric matters, which was absent in the $4 \mathrm{~d}$ case with "sconfining $\times$ s-confining". It is very interesting to further investigate the deconfinement with "s-confining $\times$ s-confining" and find a new s-confining description of the theory with two-index matters. It is also important to generalize the "s-confining $\times$ s-confining" structure to the cases with "s-confining $\times$ quantum modified constraint" or "quantum modified constraint $\times$ quantum modified constraint", which is easily performed by changing the matter contents.

The difference between the $3 \mathrm{~d}$ and $4 \mathrm{~d}$ deconfinement is that the $3 \mathrm{~d}$ deconfinement and the duality transformation affect the structure of the Coulomb branch operators. The Coulomb branch of the $3 \mathrm{~d} \mathcal{N}=2 \mathrm{SU}(N)$ gauge theory with anti-symmetric matters is very complicated and sometimes the two coordinates are required to describe it [25, 31]. In the deconfined description we also have the two Coulomb branch operators: one of them is a $\mathrm{SU}(N)$ Coulomb branch and the other one comes from a $\operatorname{Sp}(2 N)$ gauge theory. Remember that in the deconfined description discussed in this paper, the $\mathrm{SU}(N)$ part of the $\mathrm{SU} \times \mathrm{Sp}$ gauge theory is "vector-like", so we need only a single operator for describing the $\mathrm{SU}(N)$ Coulomb branch. These two Coulomb branches correctly explain the unlifted Coulomb branch of the $3 \mathrm{~d} \mathcal{N}=2 \mathrm{SU}(N)$ gauge theory with anti-symmetric matters. When one first changes the dynamics of the Sp part into the confined description, the Coulomb branch of the $\mathrm{SU}(N)$ part becomes highly complicated as explained in $[25,31]$. If we first change the $\mathrm{SU}(N)$ dynamics into the Seiberg dual or a confined one, the Coulomb branch of the Sp 
part is modified because the fermions contributing to the Sp-monopole are mesons. When we further exchange the Sp part to the dual description and obtain the second dual, the Coulomb branch of the SU part becomes complicated since the duality transformation of the Sp part introduces the two-index matters in the $\mathrm{SU}(N)$ gauge group. In $4 \mathrm{~d}$, we can think of the SU gauge group as a flavor symmetry under the Sp Seiberg duality transformation and only the matter contents change. The modification of the Coulomb branch under the duality sequence is peculiar to the $3 \mathrm{~d}$ deconfinement.

We showed the alternative way of the deconfinement, namely decoupling the Coulomb moduli. In this method we do not lift the unnecessary Coulomb branch but only decouple it from the other sector by introducing the additional matters. This is successful but it is difficult to find the decoupled direction in the dual theories. In order to deal with this problem, it would be preferable to find the decoupled degrees of freedom on the dual side, for example, by using the argument in [40], where the quantum dimensions of the operators are investigated by means of the F-maximization and the decoupled directions are studied. We can use the same technique in our example.

In this paper we checked the validity of the deconfinement with "s-confining $\times$ s-confining" by the correspondence of the moduli space and the comparison with the known dualities. Although this check is quite strong, it is worth performing the another checks. For example, we can compare the (superconformal) indices of the electric and magnetic theories by using the localization method [42-46]. Furthermore it is important to find the correct $\mathrm{U}(1)_{R}$ charge realized in the infrared comformal fixed point, which would be possible if we use the F-maximization. If the duality is correct, the infrared $\mathrm{U}(1)_{R}$ charges on the electric and magnetic sides should be identical. This would be a non-trivial check of the duality.

It is also possible to apply the $3 \mathrm{~d}$ deconfinement technique to the (supersymmetric) Chern-Simons matter theories with two-index matters. This is a simple generalization and all the story which we gave in this paper can be applied. In section 5, we studied the deconfinement of the $3 \mathrm{~d} \mathcal{N}=2 \mathrm{SU}(N)$ gauge theory with one anti-symmetric, $F$ fundamentals and $F^{\prime}=F+2 N-2$ anti-fundamentals. The deconfined theory is a vectorlike theory and its dual is given by the $\mathrm{U}(F-N) \times \mathrm{Sp}(N+K-2)$ gauge theory. It is interesting to consider the chiral version. The Seiberg duality of the chiral gauge theory is considered in $[17,47,48]$ and we can use it for finding the duality of the $3 \mathrm{~d} \mathcal{N}=2 \mathrm{SU}(N)$ gauge theory with an anti-symmetric, $F$ fundamentals and general $F^{\prime}$ anti-fundamentals. It is also possible and interesting to study the deconfinement of the adjoint matters and the symmetric matters. These are described in terms of the s-confining phases of the $(S) \mathrm{U}(N)$ and $(S) O(N)$ gauge theories respectively.

Now we have the $3 \mathrm{~d}$ and $4 \mathrm{~d}$ deconfinement methods. Then it is quite intriguing to understand the relation between them. It would be possible to dimensionally reduce the $4 \mathrm{~d}$ deconfinement description into the $3 \mathrm{~d}$ one because the deconfined description only contains the fundamental matters, so the reduction and the deformation required for obtaining the $3 \mathrm{~d}$ duality would be the same as [17]. The procedure is very simple: first, we put the $4 \mathrm{~d}$ theory on a circle and take into account the effect of the compactification. The effect mainly comes from the KK-monopoles (twisted instantons) [49], which generate the non- 
perturbative superpotential. The same step is also carried out for the dual theory. In this way we can obtain the $3 \mathrm{~d}$ or $\mathbb{R}^{3} \times \mathbb{S}^{1}$ duality with the superpotential. In order to obtain the duality without the superpotential, we need to further deform the theory by giving real masses and taking the low-energy limit. On the dual side we need to find the same deformation as the electric side. However since the global U(1) charge assignment of the matter contents is complicated in the deconfinement theory, it is difficult to find where the low-energy limit should be taken in the dual theory. Thus it is very interesting problem to find the connection of the $3 \mathrm{~d}$ and $4 \mathrm{~d}$ deconfinement methods.

In section4, we discussed the dimensional reduction of the special class of the $\mathcal{N}=1$ Seiberg-Witten curve where the $4 \mathrm{~d}$ theory only has the rank-1 Coulomb branch. But one can generally write down the Seiberg-Witten curves for the theories with also the Higgs branch. The dimensional reduction would well work for such theories and one can obtain the effective 3d superpotentials including the Higgs and Coulomb moduli from the SeibergWitten curve. We will briefly show the story of this direction. The Seiberg-Witten curve generically contains the mass or coupling dependence for the matter chiral superfields and in this sense the Higgs branch is integrated out. In the dimensional reduction of the theory whose dynamics on the Coulomb branch is described by the Seiberg-Witten curve, we can interpret the variables $x, y$ describing the curve as the dynamical fields. Integrating in the matter contribution into the superpotential by inverse Legendre transformations we obtain the correct description of the Coulomb and Higgs branches. To be more specific, let us consider the $4 \mathrm{~d} \mathcal{N}=2 \mathrm{SU}(2)$ gauge theory with $N_{f}=1$ fundamental flavors [50]. The corresponding Seiberg-Witten curve is

$$
y^{2}=x^{2}(x-u)+2 m x+1,
$$

where $m$ is a mass term for the hypermultiplet and $u:=\operatorname{tr} \phi^{2}$. By changing the variables as $x-u=: v$ [50], compactifying the theory on a circle and introducing the soft SUSY breaking term to the adjoint chiral superfield since we are interested in the dynamics of the $3 \mathrm{~d} \mathcal{N}=2$ theories, we obtain the superpotential

$$
W=\lambda\left(y^{2}-x^{2} v-2 m x-1\right)+\epsilon(x \eta-v),
$$

where $\epsilon$ is a SUSY breaking parameter, $\eta$ is a $4 \mathrm{~d}$ dynamical scale and $\lambda$ is a Langrange multiplier to impose the defining equation of the Seiberg-Witten curve. Integrating out $\lambda, y$ and $v$, we find the low-energy superpotential

$$
W=\epsilon x \eta+\epsilon \frac{1-2 m x}{x^{2}} .
$$

Note that since the effective superpotential has the linear dependence on $m$, then Legendre transforming $m$ to the meson field $M$ gives the identification $M \sim \frac{2 \epsilon}{x}$. If we rescale $x$ as $Y:=x /(2 \epsilon)$ and interpret this as the Coulomb branch operator, we can find the quantum modified constraint $M Y=1$, which is found in [7]. In this rescale and by taking the $3 \mathrm{~d}$ limit where $\eta \rightarrow 0$, the first and second terms in the superpotential are vanishing. This analysis is straightforwardly generalized to $N_{f}=2$ [50], in which the Seiberg-Witten curve 
takes

$$
y^{2}=\left(x^{2}-1\right) v+2 m_{1} m_{2} x-\left(m_{1}^{2}+m_{2}^{2}\right)
$$

in terms of the rescaled variables. We can integrate in the meson fields $M_{12}$ and $M_{34}$ and find

$$
W=-\frac{M_{12} M_{34} x}{2 \epsilon}+\cdots=-M_{12} M_{34} Y+\cdots
$$

where the ellipses are the terms vanishing in the limit with $\epsilon \rightarrow \infty$ and $\eta \rightarrow 0$. This result is beautifully consistent with the effective superpotential $W=-Y \operatorname{Pf} M$. Notice that since the above derivation relies on the integrating-in method, we may in general include other terms and in many cases we are missing new massless degrees of freedom appearing in the origin of the moduli space. The similar analyses are found in [51-54] and it would be possible to generalize the analysis to more complicated $\mathcal{N}=1$ Seiberg-Witten curves with the higher ranks. It is also worth investigating the $3 \mathrm{~d} \mathcal{N}=2 \mathrm{SU}(N) \times \mathrm{SU}(N)$ theory with bi-fundamental matters since the $4 \mathrm{~d}$ version of this theory has the Coulomb phases and we will be able to find the interesting physics also in $3 \mathrm{~d}$.

\section{Acknowledgments}

I would like to thank Ofer Aharony and Ken Intriligator for useful discussions. I am also grateful to Ashoke Sen for helpful comments.

Open Access. This article is distributed under the terms of the Creative Commons Attribution License (CC-BY 4.0), which permits any use, distribution and reproduction in any medium, provided the original author(s) and source are credited.

\section{References}

[1] N. Seiberg, Electric-magnetic duality in supersymmetric nonAbelian gauge theories, Nucl. Phys. B 435 (1995) 129 [hep-th/9411149] [INSPIRE].

[2] K.A. Intriligator, R.G. Leigh and M.J. Strassler, New examples of duality in chiral and nonchiral supersymmetric gauge theories, Nucl. Phys. B 456 (1995) 567 [hep-th/9506148] [INSPIRE].

[3] M. Berkooz, The Dual of supersymmetric $\mathrm{SU}(2 K)$ with an antisymmetric tensor and composite dualities, Nucl. Phys. B 452 (1995) 513 [hep-th/9505067] [INSPIRE].

[4] P. Pouliot, Duality in SUSY SU(N) with an antisymmetric tensor, Phys. Lett. B 367 (1996) 151 [hep-th/9510148] [INSPIRE].

[5] M.A. Luty, M. Schmaltz and J. Terning, A sequence of duals for $\mathrm{Sp}(2 N)$ supersymmetric gauge theories with adjoint matter, Phys. Rev. D 54 (1996) 7815 [hep-th/9603034] [INSPIRE].

[6] I. Affleck, J.A. Harvey and E. Witten, Instantons and (Super)Symmetry Breaking in (2+1)-Dimensions, Nucl. Phys. B 206 (1982) 413 [INSPIRE]. 
[7] O. Aharony, A. Hanany, K.A. Intriligator, N. Seiberg and M.J. Strassler, Aspects of $N=2$ supersymmetric gauge theories in three-dimensions, Nucl. Phys. B 499 (1997) 67 [hep-th/9703110] [INSPIRE].

[8] J. de Boer, K. Hori and Y. Oz, Dynamics of $N=2$ supersymmetric gauge theories in three-dimensions, Nucl. Phys. B 500 (1997) 163 [hep-th/9703100] [INSPIRE].

[9] A. Giveon and D. Kutasov, Seiberg Duality in Chern-Simons Theory, Nucl. Phys. B 812 (2009) 1 [arXiv: 0808.0360] [INSPIRE].

[10] O. Aharony, IR duality in $D=3 N=2$ supersymmetric $\operatorname{USp}\left(2 N_{c}\right)$ and $\mathrm{U}\left(N_{c}\right)$ gauge theories, Phys. Lett. B 404 (1997) 71 [hep-th/9703215] [INSPIRE].

[11] V. Niarchos, Seiberg Duality in Chern-Simons Theories with Fundamental and Adjoint Matter, JHEP 11 (2008) 001 [arXiv:0808.2771] [INSPIRE].

[12] V. Niarchos, $R$-charges, Chiral Rings and $R G$ Flows in Supersymmetric Chern-Simons-Matter Theories, JHEP 05 (2009) 054 [arXiv:0903.0435] [INSPIRE].

[13] H. Kim and J. Park, Aharony Dualities for 3d Theories with Adjoint Matter, JHEP 06 (2013) 106 [arXiv:1302.3645] [InSPIRE].

[14] J. Park and K.-J. Park, Seiberg-like Dualities for $3 d N=2$ Theories with $\mathrm{SU}(N)$ gauge group, JHEP 10 (2013) 198 [arXiv: 1305.6280] [INSPIRE].

[15] K. Intriligator and N. Seiberg, Aspects of $3 d N=2$ Chern-Simons-Matter Theories, JHEP 07 (2013) 079 [arXiv: 1305.1633] [inSPIRE].

[16] K. Intriligator, Matching $3 d N=2$ vortices and monopole operators, JHEP 10 (2014) 52 [arXiv: 1406.2638] [INSPIRE].

[17] O. Aharony, S.S. Razamat, N. Seiberg and B. Willett, $3 d$ dualities from $4 d$ dualities, JHEP 07 (2013) 149 [arXiv: 1305.3924] [INSPIRE].

[18] O. Aharony, S.S. Razamat, N. Seiberg and B. Willett, 3d dualities from 4d dualities for orthogonal groups, JHEP 08 (2013) 099 [arXiv: 1307.0511] [INSPIRE].

[19] A. Amariti, D. Forcella, C. Klare, D. Orlando and S. Reffert, The braneology of $3 D$ dualities, J. Phys. A 48 (2015) 265401 [arXiv:1501.06571] [InSPIRE].

[20] A. Amariti, D. Forcella, C. Klare, D. Orlando and S. Reffert, 4D/3D reduction of dualities: mirrors on the circle, JHEP 10 (2015) 048 [arXiv: 1504.02783] [INSPIRE].

[21] K.A. Intriligator and N. Seiberg, Phases of $N=1$ supersymmetric gauge theories in four-dimensions, Nucl. Phys. B 431 (1994) 551 [hep-th/9408155] [INSPIRE].

[22] K.A. Intriligator, R.G. Leigh and N. Seiberg, Exact superpotentials in four-dimensions, Phys. Rev. D 50 (1994) 1092 [hep-th/9403198] [INSPIRE].

[23] C. Csáki, J. Erlich, D.Z. Freedman and W. Skiba, N=1 supersymmetric product group theories in the Coulomb phase, Phys. Rev. D 56 (1997) 5209 [hep-th/9704067] [InSPIRE].

[24] N. Seiberg and E. Witten, Gauge dynamics and compactification to three-dimensions, hep-th/9607163 [INSPIRE].

[25] C. Csáki, M. Martone, Y. Shirman, P. Tanedo and J. Terning, Dynamics of 3D SUSY Gauge Theories with Antisymmetric Matter, JHEP 08 (2014) 141 [arXiv:1406.6684] [INSPIRE].

[26] A. Karch, Seiberg duality in three-dimensions, Phys. Lett. B 405 (1997) 79 [hep-th/9703172] [INSPIRE]. 
[27] C. Callias, Index Theorems on Open Spaces, Commun. Math. Phys. 62 (1978) 213 [inSPIRE].

[28] E.J. Weinberg, Fundamental Monopoles and Multi-Monopole Solutions for Arbitrary Simple Gauge Groups, Nucl. Phys. B 167 (1980) 500 [InSPIRE].

[29] E.J. Weinberg, Fundamental Monopoles in Theories With Arbitrary Symmetry Breaking, Nucl. Phys. B 203 (1982) 445 [inSPIRE].

[30] T. Hirayama and K. Yoshioka, Duality between simple group gauge theories and some applications, Phys. Rev. D 59 (1999) 105005 [hep-th/9811119] [INSPIRE].

[31] A. Amariti, C. Csáki, M. Martone and N. R.-L. Lorier, From 4D to 3D chiral theories: Dressing the monopoles, Phys. Rev. D 93 (2016) 105027 [arXiv:1506.01017] [INSPIRE].

[32] H. Murayama, Studying noncalculable models of dynamical supersymmetry breaking, Phys. Lett. B 355 (1995) 187 [hep-th/9505082] [INSPIRE].

[33] E. Poppitz and S.P. Trivedi, Some examples of chiral moduli spaces and dynamical supersymmetry breaking, Phys. Lett. B 365 (1996) 125 [hep-th/9507169] [INSPIRE].

[34] C. Csáki, M. Schmaltz and W. Skiba, A systematic approach to confinement in $N=1$ supersymmetric gauge theories, Phys. Rev. Lett. 78 (1997) 799 [hep-th/9610139] [INSPIRE].

[35] C. Csáki, M. Schmaltz and W. Skiba, Confinement in $N=1$ SUSY gauge theories and model building tools, Phys. Rev. D 55 (1997) 7840 [hep-th/9612207] [INSPIRE].

[36] C. Csáki, The confining $N=1$ supersymmetric gauge theories: A review, hep-th/9807222 [INSPIRE].

[37] C. Csáki and W. Skiba, Classification of the $N=1$ Seiberg-Witten theories, Phys. Rev. D 58 (1998) 045008 [hep-th/9801173] [INSPIRE].

[38] M. Gremm, The Coulomb branch of $N=1$ supersymmetric $\mathrm{SU}\left(N_{c}\right) \times \mathrm{SU}\left(N_{c}\right)$ gauge theories, Phys. Rev. D 57 (1998) 2537 [hep-th/9707071] [INSPIRE].

[39] O. Aharony and I. Shamir, On $O\left(N_{c}\right) D=3 N=2$ supersymmetric QCD Theories, JHEP 12 (2011) 043 [arXiv: 1109.5081] [inSPIRE].

[40] J. Lee and M. Yamazaki, Gauging and decoupling in $3 d \mathcal{N}=2$ dualities, JHEP 06 (2016) 077 [arXiv: 1603. 02283] [INSPIRE].

[41] K.A. Intriligator and N. Seiberg, Duality, monopoles, dyons, confinement and oblique confinement in supersymmetric $\mathrm{SO}\left(N_{c}\right)$ gauge theories, Nucl. Phys. B 444 (1995) 125 [hep-th/9503179] [INSPIRE].

[42] V. Pestun, Localization of gauge theory on a four-sphere and supersymmetric Wilson loops, Commun. Math. Phys. 313 (2012) 71 [arXiv:0712.2824] [INSPIRE].

[43] A. Kapustin, B. Willett and I. Yaakov, Exact Results for Wilson Loops in Superconformal Chern-Simons Theories with Matter, JHEP 03 (2010) 089 [arXiv:0909.4559] [INSPIRE].

[44] A. Kapustin, B. Willett and I. Yaakov, Nonperturbative Tests of Three-Dimensional Dualities, JHEP 10 (2010) 013 [arXiv: 1003.5694] [inSPIRE].

[45] N. Hama, K. Hosomichi and S. Lee, Notes on SUSY Gauge Theories on Three-Sphere, JHEP 03 (2011) 127 [arXiv: 1012.3512] [INSPIRE].

[46] Y. Imamura and S. Yokoyama, Index for three dimensional superconformal field theories with general R-charge assignments, JHEP 04 (2011) 007 [arXiv: 1101.0557] [INSPIRE]. 
[47] F. Benini, C. Closset and S. Cremonesi, Comments on 3d Seiberg-like dualities, JHEP 10 (2011) 075 [arXiv: 1108.5373] [INSPIRE].

[48] O. Aharony and D. Fleischer, IR Dualities in General 3d Supersymmetric $\mathrm{SU}(N)$ QCD Theories, JHEP 02 (2015) 162 [arXiv:1411.5475] [INSPIRE].

[49] K.-M. Lee and P. Yi, Monopoles and instantons on partially compactified D-branes, Phys. Rev. D 56 (1997) 3711 [hep-th/9702107] [INSPIRE].

[50] N. Seiberg and E. Witten, Monopoles, duality and chiral symmetry breaking in $N=2$ supersymmetric QCD, Nucl. Phys. B 431 (1994) 484 [hep-th/9408099] [InSPIRE].

[51] R. Boels, J. de Boer, R. Duivenvoorden and J. Wijnhout, Nonperturbative superpotentials and compactification to three-dimensions, JHEP 03 (2004) 009 [hep-th/0304061] [INSPIRE].

[52] R. Boels and J. de Boer, Classical spin chains and exact three-dimensional superpotentials, Nucl. Phys. B 715 (2005) 234 [hep-th/0411110] [InSPIRE].

[53] R. Boels, J. de Boer, R. Duivenvoorden and J. Wijnhout, Factorization of Seiberg-Witten curves and compactification to three-dimensions, JHEP 03 (2004) 010 [hep-th/0305189] [INSPIRE].

[54] N. Dorey, An elliptic superpotential for softly broken $N=4$ supersymmetric Yang-Mills theory, JHEP 07 (1999) 021 [hep-th/9906011] [INSPIRE]. 